\title{
Nano-biphasic calcium phosphate/polyvinyl alcohol composites with enhanced bioactivity for bone repair via low-temperature three-dimensional printing and loading with platelet-rich fibrin
}

This article was published in the following Dove Press journal:

International Journal of Nanomedicine

\author{
Yue Songl,* \\ Kaifeng Lin ${ }^{2, *}$ \\ Shu $\mathrm{He}^{3, *}$ \\ Chunmei Wang' \\ Shuaishuai Zhang' \\ Donglin $\mathrm{Li}^{\prime}$ \\ Jimeng Wang ${ }^{4}$ \\ Tianqing $\mathrm{CaO}^{\prime}$ \\ Long $\mathrm{Bi}^{\prime}$ \\ Guoxian Pei'
}

'Department of Orthopedics, Xijing Hospital, The Fourth Military Medical University, Xi'an, China; ${ }^{2}$ Second Department of Orthopedics and Traumatology, Fuzhou General Hospital of Nanjing Military Area Command of Chinese PLA, Fuzhou, China; ${ }^{3}$ Department of Orthopedics, Xi'an Hong Hui Hospital, Xi'an, China; ${ }^{4}$ Department of Orthopedics, The 25 I st Hospital of Chinese PLA, Zhangjiakou, China

*These authors contributed equally to this work
Correspondence: Long Bi; Guoxian Pei Department of Orthopedics, Xijing Hospital, The Fourth Military Medical University, 15 Changle West Street, Xi'an 710032, China

Tel +86 298477 I0I3; +862984773524

Email bilong@fmmu.edu.cn; pgx1954@163.com

\begin{abstract}
Background and aim: As a newly emerging three-dimensional (3D) printing technology, low-temperature robocasting can be used to fabricate geometrically complex ceramic scaffolds at low temperatures. Here, we aimed to fabricate $3 \mathrm{D}$ printed ceramic scaffolds composed of nano-biphasic calcium phosphate (BCP), polyvinyl alcohol (PVA), and platelet-rich fibrin (PRF) at a low temperature without the addition of toxic chemicals.

Methods: Corresponding nonprinted scaffolds were prepared using a freeze-drying method. Compared with the nonprinted scaffolds, the printed scaffolds had specific shapes and wellconnected internal structures.
\end{abstract}

Results: The incorporation of PRF enabled both the sustained release of bioactive factors from the scaffolds and improved biocompatibility and biological activity toward bone marrow-derived mesenchymal stem cells (BMSCs) in vitro. Additionally, the printed BCP/PVA/PRF scaffolds promoted significantly better BMSC adhesion, proliferation, and osteogenic differentiation in vitro than the printed BCP/PVA scaffolds. In vivo, the printed BCP/PVA/PRF scaffolds induced a greater extent of appropriate bone formation than the printed BCP/PVA scaffolds and nonprinted scaffolds in a critical-size segmental bone defect model in rabbits.

Conclusion: These experiments indicate that low-temperature robocasting could potentially be used to fabricate $3 \mathrm{D}$ printed $\mathrm{BCP} / \mathrm{PVA} / \mathrm{PRF}$ scaffolds with desired shapes and internal structures and incorporated bioactive factors to enhance the repair of segmental bone defects.

Keywords: three-dimensional printing, nano-biphasic calcium phosphate, polyvinyl alcohol, platelet-rich fibrin, bone substitutes, tissue engineering

\section{Introduction}

Large bone defects are frequently encountered by orthopedic surgeons in clinical practice and are great surgical challenges. Bone traumas exceeding a critical size become scarred and lose the ability to regenerate. Autologous bone grafting is considered the gold standard repair method; however, the transplantation process may lead to hemorrhage, nerve injury, and dysfunction. ${ }^{1}$ The failure of autograft is mostly due to the necrosis of grafts, which is usually caused by poor blood supply and secondary infection. To avoid these postoperative complications, the currently favored solution is to repair bone defects with inert bone graft materials (scaffolds) that simply function as a supporting structure but provide limited skeletal regeneration ability. ${ }^{2}$

The biomaterials used to fabricate scaffolds for bone repair play a key role in tissue engineering as their biophysical and biochemical characteristics affect cellular 
behavior and function. ${ }^{3}$ Numerous biomaterials are currently under development with the aim of mimicking the regulatory characteristics of the natural extracellular matrix (ECM) and ECM-bound growth factors. ${ }^{4}$ In addition, it is equally important that the technology used to prepare biomaterial scaffolds retains the maximal degree of biological activity ${ }^{5}$ and the internal pore structure facilitates the penetration of newly formed tissue. ${ }^{6}$ Indeed, the design of scaffolds with a desired shape, internal channel network, and biological activity has become an intensive area of research., ${ }^{7,8}$

Three-dimensional (3D) printing is a continually expanding, revolutionary, powerful manufacturing technique with a wide range of applications. The typical medical applications of 3D printing include preoperative planning and simulation, sharing detailed surgical information before surgery with patients, and the development of individualized implants and prostheses. ${ }^{9} 10$ Unquestionably, 3D printing represents a major opportunity for regenerative medicine ${ }^{11}$ and has been applied in attempts to address the demand for tissue engineered scaffolds, artificial tissues, and even organs suitable for transplantation. ${ }^{5}$

$3 \mathrm{D}$ printing is a broad concept that can be classified as additive manufacturing. Common additive technologies applied in medicine include selective laser sintering, ${ }^{12}$ fused deposition modeling, ${ }^{13}$ multi-jet modeling, ${ }^{14}$ stereolithography, ${ }^{15}$ powder-based printing, ${ }^{16}$ and robocasting. ${ }^{17}$ All of these techniques can be used to rapidly fabricate products with specific shapes, well-defined internal structures, and highly interconnected porosities. ${ }^{17}$

Robocasting is an additive manufacturing technique in which a thin filament of biomaterial is extruded from a nozzle, forming an object layer by layer. This technique can be used to fabricate geometrically complex ceramic scaffolds using water-based inks with minimal chemical solvent incorporation and without requiring solidification for the product to retain its shape after extrusion. ${ }^{18}$ Robocasting enables scaffolds to be printed at low temperatures, which is a major advantage over other additive manufacturing techniques. These characteristics of robocasting make it possible to incorporate bioactive molecules into scaffolds while retaining maximal levels of biological activity. ${ }^{19}$

Bone is a dynamically changing, highly vascularized tissue composed of $70 \%$ hydroxyapatite (HAp) crystals and $30 \%$ organic material by dry weight. ${ }^{20}$ In bone tissue engineering applications, bioceramics mainly function as a mechanical support and calcium store. ${ }^{21}$ Biphasic calcium phosphate (BCP) is commonly used as a material in orthopedic surgery and has two components, $\mathrm{HAp}\left(\mathrm{Ca}_{5}\left(\mathrm{PO}_{4}\right)_{3} \mathrm{OH}\right)$ and $\beta$-tricalcium phosphate $\left(\beta\right.$-TCP; $\left.\beta-\mathrm{Ca}_{3}\left(\mathrm{PO}_{4}\right)_{2}\right){ }^{22} \beta$-TCP has the advantage of having a higher solubility than HAp, which accelerates scaffold degradation. ${ }^{23}$ However, HAp is more hydrophilic than $\beta$-TCP. BCP combines the advantages of HAp and $\beta$-TCP and possesses the mixed dissolution properties of these materials. ${ }^{24}$

With respect to the adhesives required for 3D printing, polyvinyl alcohol (PVA) is a synthetic, water-soluble, wellcharacterized biocompatible polymer with viscoelastic properties comparable to those of articular cartilage. PVA has previously been used to carry and sustain the release of biologically active molecules..$^{25,26}$

Platelet-rich fibrin (PRF) is a second-generation platelet concentrate with a simplified preparation method that does not require the addition of thrombin or calcium chloride. ${ }^{27,28}$ PRF has been reported to have the potential to facilitate hemostasis, wound healing, and the differentiation of preosteoblasts due to the presence of secreted growth factors. During the process of preparing PRF, high numbers of activated platelets and leukocyte growth factors are embedded into the fibrin matrix, which are released slowly as the fibrin degrades. ${ }^{29}$ Studies have also shown that PRF facilitates osteoblast adhesion and upregulates collagen protein expression. $^{30,31}$

In this study, we fabricated an innovative 3D printed porous nano-BCP/PVA/PRF scaffold. There is little research on the combination of these three materials for bone repair applications, ${ }^{32-34}$ let alone the 3D printing of this mixture. We hypothesized that the low-temperature 3D printing of $\mathrm{BCP} /$ PVA/PRF scaffolds would preserve the biological activity of PRF and provide an innovative biomaterial for restoring segmental bone defects. The biological effects of the printed BCP/PVA/PRF scaffolds on rabbit bone marrow-derived mesenchymal stem cells (BMSCs) were assessed in vitro, and the ability of the scaffolds to promote osteogenesis was investigated in vivo using a critical-size segmental bone defect model with no self-repair capability in the rabbit radius. We suggest that low temperature 3D printed BCP/PVA/PRF scaffolds may represent a promising new approach to overcome the significant hurdles that currently prevent successful segmental bone defect and bone nonunion repair in clinical practice.

\section{Methods}

\section{Fabrication of 3D printed BCP/PVA and BCP/PVA/PRF scaffolds}

The PRF preparation procedure followed a previously published protocol. ${ }^{35}$ Ear venous blood $(5 \mathrm{~mL})$ was collected without anticoagulation from the $36 \mathrm{New}$ Zealand White rabbits (male, 12 weeks old, average weight $\sim 2.5 \mathrm{~kg}$ ) used for the in vivo critical-size bone defect study. All animal procedures 
were approved by the Institutional Animal Care Committee of The Fourth Military Medical University and conducted in strict accordance with the Guidelines for the Care and Use of Laboratory Animals issued by the Chinese Council on Animal Research and Animal Care. The venous blood was immediately transferred to $10 \mathrm{~mL}$ sterile glass centrifuge tubes and centrifuged at $400 \times g$ for $10 \mathrm{~min}$; a fibrin clot formed in the middle of the tube between the red corpuscles at the bottom and acellular plasma at the top. A resistant autologous fibrin membrane was obtained by squeezing the serum out from the clot using sterile gauze, and then, the membrane was cut into fragments. Then, the PRF fragments were stored at $-80^{\circ} \mathrm{C}$. The above PRF preparation procedure was performed the day before the BCP/PVA slurry preparation.

Nano-BCP ceramic powder consisting of $60 \%$ HAp and $40 \% \beta$-TCP with an average grain size of $100 \mathrm{~nm}$ was purchased from Emperor Nano Materials Company (Nanjing, China). PVA was purchased from Sigma-Aldrich Co. (St Louis, MO, USA) (molecular weight 85,000-124,000 Da and degree of hydrolysis $>99 \%$ ). Briefly, $8 \mathrm{~g}$ of PVA was dissolved in $42 \mathrm{~mL}$ of deionized water at $95^{\circ} \mathrm{C}$ to prepare a $16 \mathrm{wt} \%$ PVA solution. After cooling to $4^{\circ} \mathrm{C}, 8 \mathrm{~g}$ of $\mathrm{BCP}$ powder was added to $8 \mathrm{~mL}$ of the $16 \mathrm{wt} \%$ PVA solution (powder/liquid mass-to-volume ratio 1:1). Then, PRF granules prepared from $5 \mathrm{~mL}$ of venous blood were added and the mixture was stirred at $4{ }^{\circ} \mathrm{C}$ until the $\mathrm{BCP} / \mathrm{PVA} / \mathrm{PRF}$ paste was homogeneous.

The 3D printing was performed using a filament-free printing (FFP) printer (PC Printer; Particle Cloud Biotechnology, Xi'an, China). A flow chart of the 3D printing process is presented in Figure 1. The BCP/PVA/PRF paste was transferred into a plotting cartridge, and the bubbles produced by stirring were removed under vacuum. One end of the cartridge was mounted on the 3D printer port, and the other end was connected to the air compressor. The STL file, which was designed in advance, was loaded onto the Repetier Host Software and converted into G-code. The diameter of nozzle was $600 \mu \mathrm{m}$, and the migration velocity was $10 \mathrm{~mm} / \mathrm{s}$. The paste was extruded through the nozzle by the propeller, with the working table system making a synthetic motion along the $x-y$-axes and the nozzle moving along the $z$-axis. The slice thickness was set to $200 \mu \mathrm{m}$. An XXYY laminated design (each slice was repeated twice) was adopted to increase the porosity
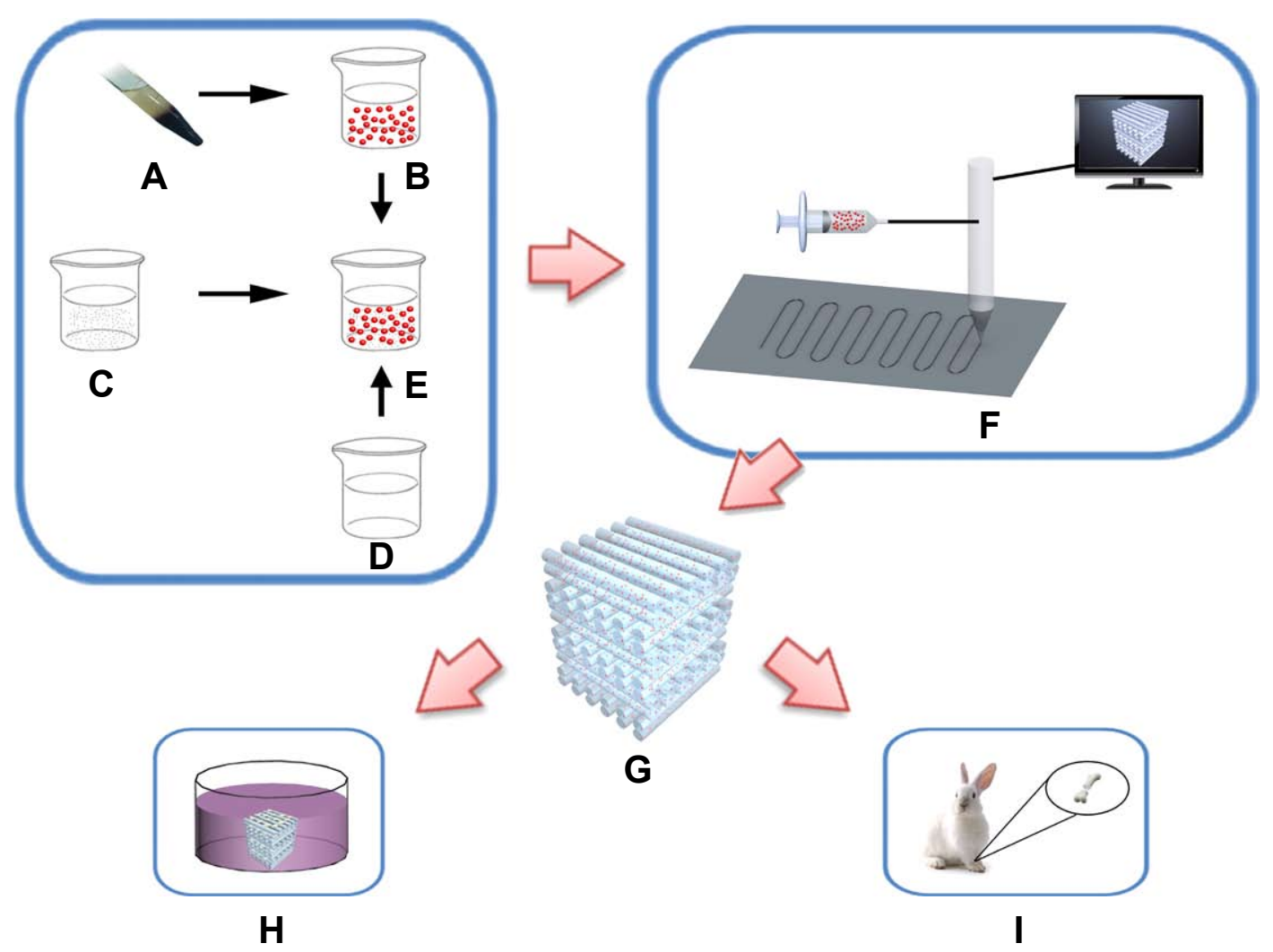

Figure I Schematic representation illustrating production and testing of the 3D printed scaffolds.

Notes: PRF clots were prepared (A), the serum was squeezed out, and the PRF membrane was cut into granules (B). Nano-BCP (C), I6 wt\% PVA solution (D), and PRF granules $(\mathbf{B})$ were mixed to prepare BCP/PVA/PRF paste $(\mathbf{E})$. Illustration of the 3D printer and printing process (F) used to create the printed scaffolds $(\mathbf{G})$ which were subjected to in vitro experiments with bone marrow mesenchymal stem cells $(\mathbf{H})$ and tested in vivo in a critical-size bone defect model in the rabbit radius $(\mathbf{I})$. Abbreviations: BCP, biphasic calcium phosphate; 3D, three-dimensional; PVA, polyvinyl alcohol; PRF, platelet-rich fibrin. 
and connectivity rate. The ambient temperature was set to $\sim 4^{\circ} \mathrm{C}$ to retain maximal cytokine activity. After printing, the scaffolds were frozen at $-80^{\circ} \mathrm{C}$ overnight. To prepare nonprinted scaffolds, the paste was cast into molds that were the same size as the printed scaffolds and solidified at $-80^{\circ} \mathrm{C}$.

The following four groups of scaffolds were prepared: nonprinted BCP/PVA scaffolds, printed BCP/PVA scaffolds, nonprinted $\mathrm{BCP} / \mathrm{PVA} / \mathrm{PRF}$ scaffolds, and printed $\mathrm{BCP} /$ PVA/PRF scaffolds. All scaffolds were lyophilized using a vacuum freeze-drying machine (Alpha 1-2 LD plus; Christ, Osterode, Germany).

\section{Characterization of the scaffolds} Microstructural analysis

The scaffolds were immersed in phosphate-buffered saline (PBS) for $24 \mathrm{~h}$, successively dehydrated in 70, 90, 95, and $100 \%$ ethanol for $15 \mathrm{~min}$, and then dried under vacuum. After coating the samples with gold, the surface and crosssectional morphologies were observed via scanning electron microscopy (SEM) using a Hitachi S-4800 system (Hitachi, Tokyo, Japan).

\section{Hydrophilic properties}

Hydrophilicity was measured using a contact angle instrument (Kruss, Hamburg, Germany), which employs an optical system to capture a photo of a drop of ultrapure water on the scaffold via the static sessile drop method $(n=6)$. The contact angle is the angle between the liquid-solid interface and the liquid-vapor interface. ${ }^{36}$

\section{Porosity}

The porosity of the scaffolds was measured using a highresolution micro-computed tomography (micro-CT) analysis system (Yxlon) with VGStudio MAX software (Volume Graphics, Heidelberg, Germany). Porosity was defined as the ratio between the pore volume and bulk volume. A volume of $10 \times 10 \times 3 \mathrm{~mm}$ was chosen as the region of interest $(n=6)$, and a threshold contrast method was used to distinguish the pore volume from the scaffold volume.

\section{Surface roughness}

Surface roughness was determined using a surface roughness tester (TR240; Beijing TIME Group, Inc., Beijing, China) and the supporting software $(n=3)$. The scanning area was $200 \times 200 \mu \mathrm{m}$, and the scanning speed was $2 \mu \mathrm{m} / \mathrm{s}$.

\section{Compressive modulus}

Cuboidal scaffolds $(\mathrm{n}=3 ; 10 \times 10 \times 3 \mathrm{~mm})$ were soaked in PBS for $24 \mathrm{~h}$ at $37^{\circ} \mathrm{C}$ to simulate in vivo conditions and were then analyzed using a Model 858 Material Testing System (MTS Systems, Eden Prairie, MN, USA) at a deformation rate of $0.005 \mathrm{~mm} / \mathrm{s}$. The compressive modulus was then obtained from the tangent slope of the stress-strain curve in the linear strain range where the material follows Hooke's law. ${ }^{37}$

\section{Biological assessment of the scaffolds Quantification of growth factor release}

The concentrations of growth factors released from the nonprinted and printed BCP/PVA/PRF scaffolds $(10 \times 10 \times$ $3 \mathrm{~mm}$ ) over 21 days were measured using commercial enzyme-linked immunosorbent assay (ELISA) kits (R\&D Systems, Inc., Minneapolis, MN, USA) following the manufacturer's instructions. The growth factors assessed were vascular endothelial growth factor (VEGF), plateletderived growth factor-AB (PDGF-AB), transforming growth factor- $\beta 1$ (TGF- $\beta 1$ ), and insulin-like growth factor-1 (IGF-1). ${ }^{38}$ The nonprinted and printed scaffolds were sterilized and placed in six-well plates; then, $2 \mathrm{~mL}$ of PBS (HyClone, Logan, UT, USA) was added to each well for incubation at $37^{\circ} \mathrm{C}$ in a $5 \%$ humidified $\mathrm{CO}_{2}$ incubator. The PBS was collected and replaced with the same amount of PBS every $24 \mathrm{~h}$ for 21 days. The PBS samples were stored at $-80^{\circ} \mathrm{C}$ and assessed in parallel.

\section{Isolation and culture of BMSCs}

Five 4-week-old male New Zealand White rabbits were anesthetized, and bone marrow was aspirated from the femoral condyle using sterilized $16 \mathrm{G}$ syringe needles that had undergone anticoagulation treatment with $1,000 \mathrm{U}$ heparin. The cells were cultured in $25 \mathrm{~cm}^{2}$ cell culture flasks in $90 \% \alpha$-minimum essential medium ( $\alpha$-MEM; HyClone) containing 10\% fetal bovine serum (Gibco, a product line of Thermo Fisher Scientific, Waltham, MA, USA), $100 \mathrm{IU} / \mathrm{mL}$ penicillin, and $100 \mu \mathrm{g} / \mathrm{mL}$ streptomycin at $37^{\circ} \mathrm{C}$ in a $5 \%$ humidified $\mathrm{CO}_{2}$ incubator. Adherent cells were passaged after $48 \mathrm{~h}$. The media were changed every 2-3 days, and the BMSCs were passaged upon reaching $80 \%$ confluence. Third-passage cells were used for biological assessments.

\section{Seeding BMSCs on the scaffolds}

The seeding procedure was performed as previously described. ${ }^{19}$ Scaffolds $(10 \times 10 \times 3 \mathrm{~mm})$ were sterilized, soaked in $\alpha$-MEM for $24 \mathrm{~h}$ before use, and placed in six-well plates; then, $3 \times 10^{5}$ cells $/ \mathrm{cm}^{3}$ were seeded onto the scaffolds in $2 \mathrm{~mL}$ of culture medium, and the plates were incubated at $37^{\circ} \mathrm{C}$. The media were changed every 3 days. 


\section{Cellular compatibility of the scaffolds}

Scaffolds were soaked in $2 \mathrm{~mL}$ of cell culture media in sixwell plates for $48 \mathrm{~h}$. BMSCs were seeded in 96-well plates at a density of $1 \times 10^{4}$ cells/well and allowed to adhere for $8 \mathrm{~h}$. Then, the cell culture media were replaced with the media in which the scaffolds had been soaked, and the BMSCs were cultured for $24 \mathrm{~h}$. Untreated cell culture media were added to the wells in the control group.

Cell numbers were assessed using Cell Counting Kit-8 (CCK-8; Dojindo, Kumamoto, Japan). Briefly, $10 \mu \mathrm{L}$ of CCK-8 solution was added to each well and the plates were incubated for $4 \mathrm{~h}$. The absorbance values were read at $450 \mathrm{~nm}$ (n=3) using a microplate reader (Wellscan MK3; Labsystems Dragon, Bucharest, Romania).

For the apoptosis assays, BMSCs were cultured on the scaffolds for $24 \mathrm{~h}$ and then trypsinized. The resulting cell suspension was centrifuged. BMSCs seeded on untreated culture dishes were prepared as a control. The cells were stained using an Annexin V-FITC/PI Apoptosis Detection Kit (Liankebio Company, Hangzhou, China) following the manufacturer's instructions and analyzed using a FACScan Flow Cytometry system ( $\mathrm{n}=3)$.

\section{Cell adhesion and proliferation}

At $8 \mathrm{~h}$ after being seeded on the scaffolds, BMSCs were digested from the scaffolds and counted using an Automated Cell Counter (TC-10; Bio-Rad Laboratories Inc., Hercules, CA, USA). Seeding efficiency was calculated as the number of cells attached to the scaffold divided by the total number of cells seeded.

Immunofluorescent staining for F-actin, vinculin, and nuclei was employed to assess BMSC adhesion. At $24 \mathrm{~h}$ after seeding, the cell/scaffold constructs were washed with PBS and fixed with $4.0 \%(\mathrm{w} / \mathrm{v})$ paraformaldehyde for $20 \mathrm{~min}$; then, the cells were permeabilized in $0.1 \%$ Triton X-100 for $5 \mathrm{~min}$ and incubated in $1 \%(\mathrm{w} / \mathrm{v})$ bovine serum albumin for $30 \mathrm{~min}$ to block nonspecific binding. Subsequently, the samples were incubated with anti-vinculin antibody (1:15 with PBS; AbD Serotec, Kidlington, UK) overnight at $4{ }^{\circ} \mathrm{C}$, rinsed three times in PBS for $5 \mathrm{~min}$, incubated with FITC-labeled goat anti-mouse IgG antibody (1:10 with PBS; CWBiotech, Beijing, China) for $4 \mathrm{~h}$ at room temperature in the dark, washed three times with PBS, incubated with rhodamine-phalloidin for $30 \mathrm{~min}$ in the dark (3:500 with PBS; Thermo Fisher Scientific), and washed three times with PBS. Finally, the samples were incubated with the nuclear stain 4',6-diamidino-2-phenylindole (SigmaAldrich Co.) for 5 min and washed three times with PBS. Then, the fluorescence intensity of vinculin (which reflects the adherent state of BMSCs) was measured and analyzed using the Image-Pro-Plus 6.0 software (Media Cybernetics, Rockville, MD, USA). ${ }^{19}$ BMSCs seeded on cell culture plates were processed as the control group.

Furthermore, the CCK-8 assay was used to quantitatively evaluate cell proliferation kinetics on the internal surfaces of the scaffolds on days 1, 4, 7, and 11 after seeding $(n=3)$ and the morphology of the cell/scaffold constructs was observed by SEM at 1, 4, and 7 days after seeding.

\section{Effect of scaffolds on the osteogenic differentiation of BMSCs}

Alkaline phosphatase (ALP) activity is considered as an early marker of osteogenesis ${ }^{39}$ and was measured using an ALP assay kit (Beyotime, Jiangshu, China) according to the manufacturer's protocol $(\mathrm{n}=3)$. After seeding BMSCs on the scaffolds, the cell culture media were replaced with osteogenic induction media (Cyagen Biosciences, Inc., Santa Clara, CA, USA) and ALP activity was quantified on days 7 and 14. Briefly, the cells on the scaffolds were collected and permeabilized by incubation in $0.1 \%$ Triton X-100 solution followed by sonication. The suspension was centrifuged, and the cell supernatant was reacted with $p$-nitrophenyl phenol (pNPP) solution for $30 \mathrm{~min}$. The rate of $p$-nitrophenol (pNP) production is directly proportional to ALP activity; ALP activity was normalized to the total protein content as determined using a BCA protein kit (Beyotime).

The mRNA expression levels of Alp, collagen type I alpha 1 (CollA1), osteopontin (Opn), runt-related transcription factor-2 (Runx-2), and glyceraldehyde-3-phosphate dehydrogenase (Gapdh) were measured at 7 and 14 days after seeding. Briefly, total mRNA was isolated from the cell/scaffold constructs using the TRIzol Reagent (Invitrogen Life Technologies, a product line of Thermo Fisher Scientific) and first strand cDNA was synthesized using the PrimeScript RT Master Mix (Takara Biotechnology Co., Dalian, China). Primers (Table 1) were designed using published gene sequences (National Center for Biotechnology Information and PubMed). Quantitative real-time polymerase chain reaction was performed using the SYBR Premix Ex Taq II (Takara Biotechnology Co.) on a CFX96 PCR System (Bio-Rad Laboratories Inc.). Gapdh was used as a housekeeping gene. ${ }^{40}$

\section{In vivo evaluation of bone healing in a critical-size bone defect model in the rabbit radius \\ Scaffold implantation}

The osteoconductivity and osteoinductivity of the scaffolds were assessed in vivo using a critical-size bone defect model in the rabbit radius. BMSCs were seeded onto sterilized 
Table I Primer sequences

\begin{tabular}{lll}
\hline Gene & Forward primer sequence $\mathbf{( 5}^{\prime} \mathbf{-} \mathbf{3}^{\prime} \mathbf{)}$ & Reverse primer sequence $\mathbf{( \mathbf { 5 } ^ { \prime } \mathbf { - 3 } \mathbf { \prime } )}$ \\
\hline Alp & GGCACAAGCACTCCCACTTT & TGGTCAATCCTGCCTCCTTC \\
Col-IAI & AAGAACGGAGATGACGGAGAAG & GCACCATCCAAACCACTGAA \\
Opn & CACCGCAGAATGCTATGTCC & GTGGTCATCGTCCTCATCCTC \\
Runx-2 & GCAGGAGGAAAATGAGCAAAG & GTGAGTGAGCAGAGCCGAGA \\
Gapdh & CCACTTTGTGAAGCTCATTTCCT & TCGTCCTCCTCTGGTGCTCT \\
\hline
\end{tabular}

scaffolds with identical shapes and sizes $(L=15 \mathrm{~mm}, W=3 \mathrm{~mm}$, $H=3 \mathrm{~mm})$ and cultured in vitro for 2 weeks $(\mathrm{n}=9)$. The time from the PRF preparation and cutting to its implantation within the scaffold into the rabbit was 17 days. Twelve-weekold New Zealand White rabbits (average weight $\sim 3 \mathrm{~kg}, \mathrm{n}=9$ ) were anesthetized using xylazine hydrochloride $(4 \mathrm{mg} / \mathrm{kg})$ and $2 \%(\mathrm{w} / \mathrm{v})$ pentobarbital $(30 \mathrm{mg} / \mathrm{kg}$ ) (both from Rongbai Biological Technology Co., Ltd, Shanghai, China) for the duration of surgery. The surgical procedure was performed as described in previously published protocols $\mathrm{s}^{41,42}$ and was approved by the Animal Care Committee of The Fourth Military Medical University.

After disinfection with povidone iodine and 70\% alcohol, the surgical site was draped with sterile towels, and the medial surface of the radius was surgically exposed. A segmental defect $(15 \mathrm{~mm})$ was created using a miniature orthopedic drill under saline irrigation. The bone defect was filled with the cell/scaffold construct, and the soft tissue was sutured using 3.0 sutures. No scaffolds were implanted into the control group $(n=9)$. The rabbits received perioperative antibiotics (50 kU/kg penicillin) by intramuscular injection and postoperative medical care.

After 4, 8, and 12 weeks, rabbits were euthanized with an overdose of ketamine hydrochloride (Hengrui Medicine Co., Ltd, Jiangsu, China). For fluorescence double staining, three rabbits from each group received an intramuscular injection of tetracycline ( $80 \mathrm{mg} / \mathrm{kg}$; Sigma-Aldrich Co.) 14 days and calcein $(8 \mathrm{mg} / \mathrm{kg}$; Sigma-Aldrich Co.) 3 days before euthanasia at 8 weeks after surgery.

\section{Micro-CT imaging and 3D reconstruction}

The radius bones were dissected, fixed in $80 \%$ ethanol, and analyzed using a micro-CT scanning system (); images were reconstructed using VGStudio MAX software (Volume Graphics, Heidelberg, Germany). The X-ray tube voltage was $80 \mathrm{kV}$, and the current was $200 \mathrm{~mA} ; 450$ micro-CT slices were acquired for each sample.

The bone volume/total volume (BV/TV) fraction was calculated as the volume of newly formed bone divided by the total volume of the region of interest. The region of interest selection was based on the initial $15 \mathrm{~mm}$ surgical defect. The RSV/SV was used to assess the rate of scaffold degradation; RSV is the residual scaffold volume, and SV is the total volume of the scaffold before implantation.

\section{Bone histological staining}

New bone formation was assessed using Van Gieson's staining, as previously described. ${ }^{43}$ The samples were dehydrated in an ascending ethanol series, cleared in xylene, embedded in methyl methacrylate (MMA), and cut into $200 \mu \mathrm{m}$ thick sections using a modified interlocked diamond saw (Microtome; Leica Microsystems, Wetzlar, Germany). The slices were pasted onto plastic histology slides and polished to eliminate scratches. The sections prepared from rabbits injected with tetracycline and calcein were observed using a fluorescence microscope to calculate the mineral apposition rate (MAR), which is defined as the ratio of the mean distance between the tetracycline fluorescent strips (yellow) and calcein fluorescent strips (green) with the dosing interval (10 days). Finally, the sections were stained with $1.2 \%$ trinitrophenol and $1 \%$ acid fuchsin and visualized using an optical microscope (DM6000B; Leica Microsystems).

\section{Statistical analysis}

The results are expressed as the mean \pm standard deviation $(\bar{x} \pm$ SD) and were analyzed using SPSS 14.0 (SPSS Inc., Chicago, IL, USA) or the GraphPad Prism 5 software (GraphPad Software, Inc., La Jolla, CA, USA). Differences between experimental groups were assessed using one-way analysis of variance followed by the post hoc Bonferroni correction. $P<0.05$ was considered statistically significant.

\section{Results}

\section{Fabrication and physicochemical characterization of 3D printed BCP/PVA/PRF scaffolds}

A novel 3D printing approach was employed to fabricate $\mathrm{BCP} / \mathrm{PVA}$ and BCP/PVA/PRF scaffolds under lowtemperature conditions in order to preserve the activity of the cytokines incorporated in PRF; nonprinted BCP/PVA and 
$\mathrm{BCP} / \mathrm{PVA} / \mathrm{PRF}$ scaffolds were generated as comparisons. The ability of the scaffolds to promote osteogenesis was assessed both in vitro and in vivo (Figure 1).

Gross observations revealed that the printed scaffolds had a regular pore structure and high pore connectivity
(Figure 2A and B), which could promote the ingrowth of BMSCs and endothelial cells and the transport of nutrients and metabolic waste. Integrative SEM analyses of the microstructure and surface morphology of the scaffolds are shown in Figure 2C-F, and the internal microstructures of the
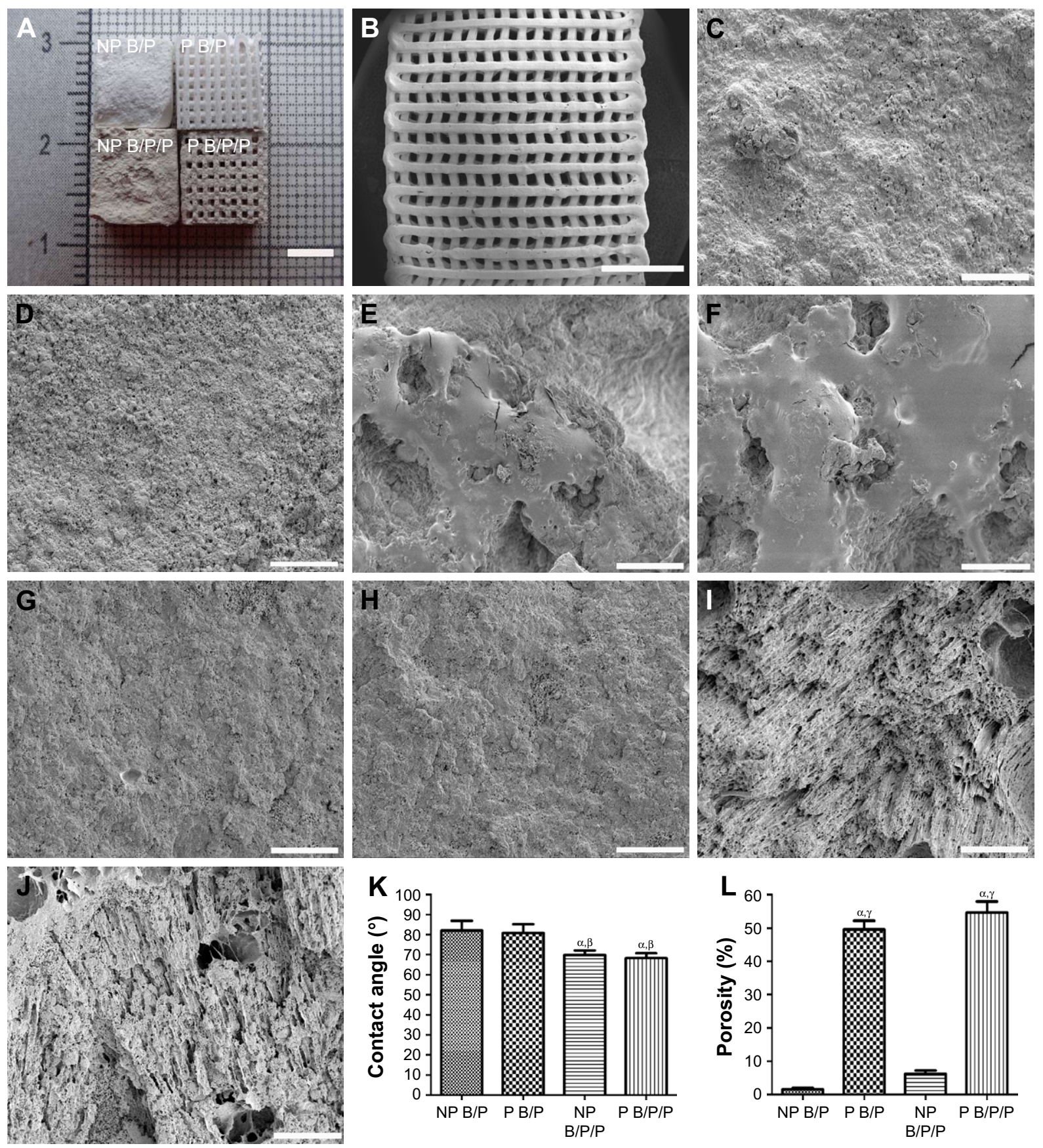

Figure 2 Macroscopic and microscopic structures, hydrophobicity, and porosity of the scaffolds.

Notes: Gross appearance of the following four types of scaffold: NP B/P scaffolds, P B/P scaffolds, NP B/P/P scaffolds, and P B/P/P scaffolds (A). The P B/P/P scaffolds had a higher porosity and higher connectivity rate $(\mathbf{B})$; scale bar: $5 \mathrm{~mm}$. Scanning electron microscopy observations of the surface morphology of NP B/P scaffolds (C), $P$ B/P scaffolds (D), NP B/P/P scaffolds (E), and P B/P/P scaffolds (F). Scanning electron microscopy observations of the internal microstructure of NP B/P scaffolds (G), $P$ B/P scaffolds (H), NP B/P/P scaffolds (I), and P B/P/P scaffolds (J); scale bars: $50 \mu \mathrm{m}$. (K) Water contact angle determination. (L) Porosity measurements. Results are mean \pm standard deviation $(\chi \pm S D), n=6 ;{ }^{\alpha} P<0.05$ vs NP B/P scaffolds; ${ }^{\beta} P<0.05$ vs $P$ B/P scaffolds; $\gamma P<0.05$ vs NP B/P/P scaffolds.

Abbreviations: NP B/P, nonprinted biphasic calcium phosphate/polyvinyl alcohol; NP B/P/P, nonprinted biphasic calcium phosphate/polyvinyl alcohol/platelet-rich fibrin; $\mathrm{P} B / \mathrm{P}$, printed biphasic calcium phosphate/polyvinyl alcohol; P B/P/P, printed biphasic calcium phosphate/polyvinyl alcohol/platelet-rich fibrin. 
scaffolds are displayed in Figure 2G-J. PRF incorporation appeared to increase the surface roughness of the scaffolds, which may promote cellular adhesion. ${ }^{44}$ The nonprinted and printed $\mathrm{BCP} / \mathrm{PVA} / \mathrm{PRF}$ scaffolds had lower contact angles than the nonprinted and printed BCP/PVA scaffolds, respectively $(P<0.05$; Figure $2 \mathrm{~K})$, demonstrating that the incorporation of PRF improved the hydrophilicity of the scaffolds. The 3D printed BCP/PVA and BCP/PVA/PRF scaffolds (49.6 and 54.7\%) had significantly higher total porosities than the nonprinted $\mathrm{BCP} / \mathrm{PVA}$ and $\mathrm{BCP} / \mathrm{PVA} / \mathrm{PRF}$ scaffolds ( 1.5 and $6.1 \%, P<0.05)$, respectively (Figure $2 \mathrm{~L}$ ), confirming that the $3 \mathrm{D}$ printing process increased the porosity of the scaffolds, which may enhance cellular penetration and distribution to facilitate vascularization.
Consistent with the SEM observations (Figure 2C-F), quantitative analysis revealed that the surface roughness of the nonprinted BCP/PVA/PRF scaffolds was threefold higher than that of the nonprinted BCP/PVA scaffolds $(P<0.05$; Figure $3 \mathrm{~A}$ ) and that the surface roughness of the $3 \mathrm{D}$ printed $\mathrm{BCP} / \mathrm{PVA} / \mathrm{PRF}$ scaffolds was 2.5-fold higher than that of the $3 \mathrm{D}$ printed $\mathrm{BCP} / \mathrm{PVA}$ scaffolds $(P<0.05)$, confirming that the incorporation of PRF significantly improved the surface roughness.

The printed BCP/PVA and BCP/PVA/PRF scaffolds had significantly lower compressive moduli values than the nonprinted $\mathrm{BCP} / \mathrm{PVA}$ and $\mathrm{BCP} / \mathrm{PVA} / \mathrm{PRF}$ scaffolds, respectively (both $P<0.05$; Figure 3B), indicating that the $3 \mathrm{D}$ printing process decreased the strength of the scaffolds.
A
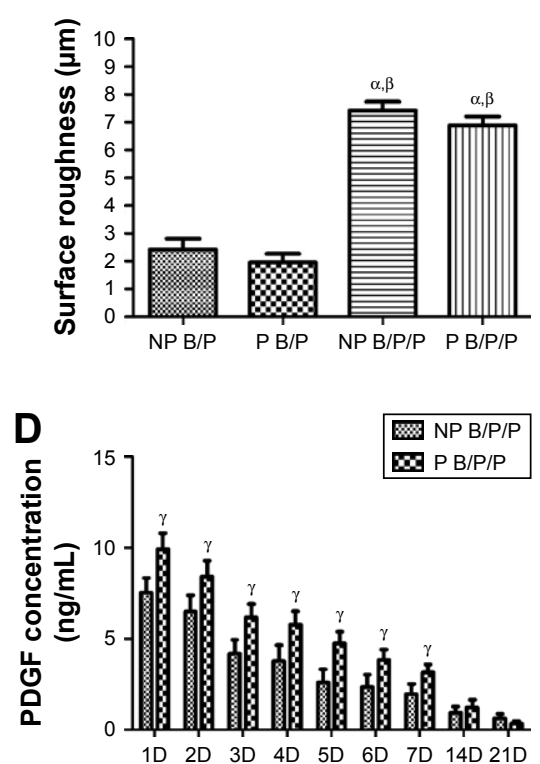

G

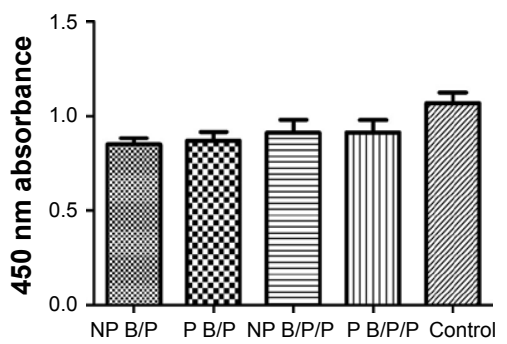

B

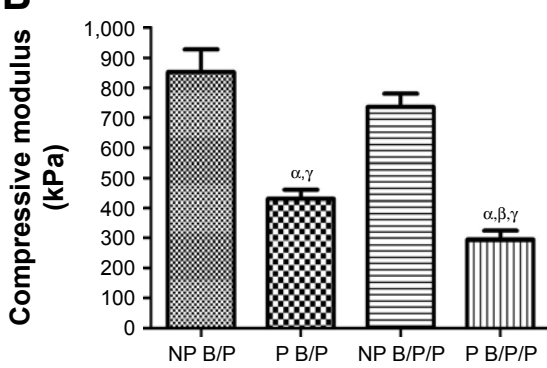

E

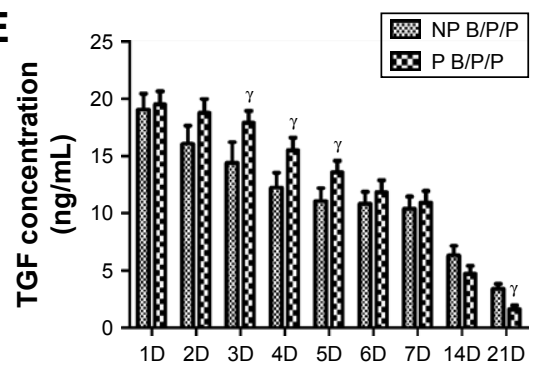

H

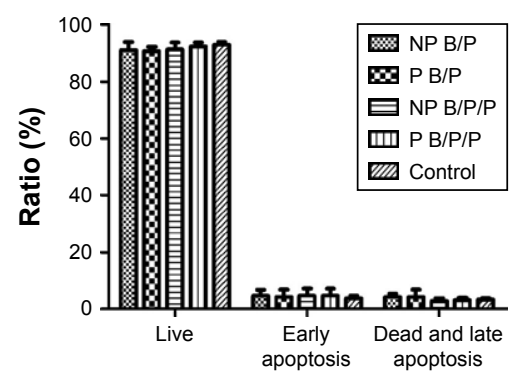

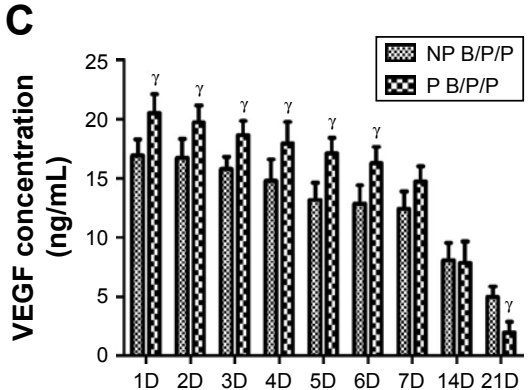

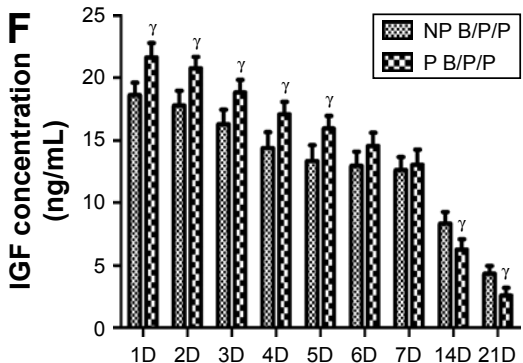

I

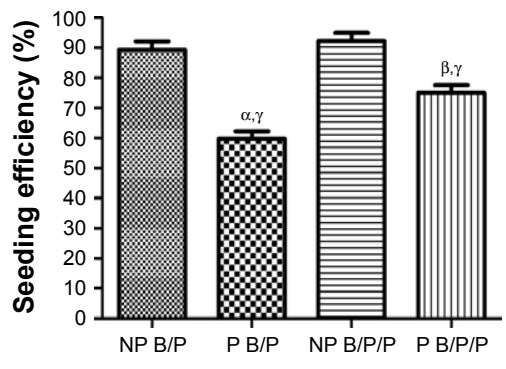

Figure 3 Assessment of the biocompatibility of the NP B/P scaffolds, P B/P scaffolds, NP B/P/P scaffolds, and P B/P/P scaffolds.

Notes: (A) Surface roughness measurements. (B) Compressive modulus calculations. Levels of growth factors released from single scaffolds into culture media over $2 \mathrm{I}$ days: VEGF (C), PDGF (D), TGF (E), and IGF (F). (G) Cellular toxicity of media in which scaffolds were soaked for $24 \mathrm{~h}$ toward BMSCs, as assessed using the CCK-8 assay. (H) Proportions of live and apoptotic BMSCs at $24 \mathrm{~h}$ after seeding on scaffolds, as assessed using the Annexin V-FITC/PI assay. (I) Seeding efficiency of BMSCs on scaffolds, as assessed by cell counting. Results are mean \pm standard deviation $(\chi \pm S D), n=3 ;{ }^{\alpha} P<0.05$ vs NP B/P scaffolds; ${ }^{\beta} P<0.05$ vs $P$ B/P scaffolds; ${ }^{\gamma P}<0.05$ vs $N P B / P / P$ scaffolds. Abbreviations: BMSCs, bone marrow-derived mesenchymal stem cells; CCK-8, Cell Counting Kit-8; IGF, insulin-like growth factor; NP B/P, nonprinted biphasic calcium phosphate/polyvinyl alcohol; NP B/P/P, nonprinted biphasic calcium phosphate/polyvinyl alcohol/platelet-rich fibrin; P B/P, printed biphasic calcium phosphate/polyvinyl alcohol; P B/P/P, printed biphasic calcium phosphate/polyvinyl alcohol/platelet-rich fibrin; PDGF, platelet-derived growth factor; VEGF, vascular endothelial growth factor; TGF, transforming growth factor. 


\section{Quantification of growth factor release from BCP/PVA/PRF scaffolds}

We quantified the levels of growth factors released by the nonprinted and printed $\mathrm{BCP} / \mathrm{PVA} / \mathrm{PRF}$ scaffolds into $\mathrm{PBS}$ over 21 days. As shown in Figure 3C-F, VEGF, PDGF, $\mathrm{TGF}$, and IGF were released from both the printed and nonprinted BCP/PVA/PRF scaffolds, although the levels decreased over time, which is in agreement with the findings of Zhao et $\mathrm{al}^{27}$ and Jayasree et $\mathrm{al}^{45}$ In general, the printed $\mathrm{BCP} / \mathrm{PVA} / \mathrm{PRF}$ scaffolds released significantly higher levels of growth factors in the early stages and significantly lower levels at 14 and 21 days than the nonprinted BCP/PVA/ PRF scaffolds.

\section{Biocompatibility of the scaffolds}

BMSCs were successfully isolated from the aspirated bone marrow samples; third-passage BMSCs were used for all experiments. The scaffolds were soaked in cell culture media for $24 \mathrm{~h}$. The cytotoxicity of the scaffold-conditioned media toward BMSCs was not significantly different from that of untreated media; none of the scaffold-conditioned media samples induced acute cytotoxicity ( $P>0.05$; Figure $3 \mathrm{G})$.

Furthermore, the apoptosis assay revealed that BMSCs seeded on the scaffolds did not undergo apoptosis or cell death $(P>0.05$; Figure $3 \mathrm{H})$. Moreover, the live/dead cell ratios were not significantly different between the control BMSCs seeded on cell culture dishes and the cells seeded on any type of scaffold ( $P>0.05$; Figure 3I).

\section{Cell adhesion and proliferation activity on the scaffolds}

Cell seeding efficiency was calculated as the number of BMSCs digested from the scaffolds divided by the initial number of cells seeded. As shown in Figure 3I, the seeding efficiency of the printed BCP/PVA scaffolds was significantly lower than that of the nonprinted $\mathrm{BCP} / \mathrm{PVA}$ scaffolds $(P<0.05)$. Moreover, the seeding efficiency of the printed $\mathrm{BCP} / \mathrm{PVA} / \mathrm{PRF}$ scaffolds was lower than that of the nonprinted $\mathrm{BCP} / \mathrm{PVA} / \mathrm{PRF}$ scaffolds $(P<0.05)$ but significantly higher than that of the printed $\mathrm{BCP} / \mathrm{PVA}$ scaffolds $(P<0.05)$.

SEM analysis was used to visualize the adhesion of the BMSCs to the printed $\mathrm{BCP} / \mathrm{PVA}$ and $\mathrm{BCP} / \mathrm{PVA} / \mathrm{PRF}$ scaffolds at $24 \mathrm{~h}$ after seeding (Figure $4 \mathrm{~A}$ and $\mathrm{B}$ ). The seeded BMSCs extended long and abundant pseudopodia and adhered firmly to the scaffolds. However, the BMSCs seeded on the printed BCP/PVA/PRF scaffolds seemed to spread over larger areas. Immunofluorescent staining for
F-action, vinculin, and nuclei in the BMSCs cultured in the scaffolds and cell culture plates was performed at $24 \mathrm{~h}$ after seeding. Vinculin is a cytoskeletal protein that localizes to focal adhesion plaques at the junctions between cells and the ECM. ${ }^{46}$ The BMSCs seeded to all scaffolds exhibited good attachment and spreading (Figure 4D).

To quantitatively evaluate the state of adhesion, the integral optical density (IOD) values for vinculin were calculated for the cells on each type of scaffold (Figure 4C). BMSCs on the printed $\mathrm{BCP} / \mathrm{PVA}$ scaffolds had a lower mean IOD value than cells on the printed BCP/PVA/PRF scaffolds (11.25 \pm 2.07 vs $24.32 \pm 3.43 ; P=0.0048)$, and BMSCs on the nonprinted BCP/PVA scaffolds had a lower mean IOD than cells on the nonprinted BCP/PVA/PRF scaffolds (11.09 \pm 1.76 vs $24.20 \pm 1.98 ; P=0.001)$. The mean IOD values of cells seeded on the nonprinted and printed BCP/PVA/PRF scaffolds were higher than those of cells seeded on the cell culture plates, while BMSCs seeded on the cell culture plates had higher mean IOD values than cells seeded on the nonprinted and printed $\mathrm{BCP} / \mathrm{PVA}$ scaffolds; however, these differences were not statistically significant.

The cell proliferation rate was higher on the printed $\mathrm{BCP} /$ PVA/PRF scaffolds than the printed BCP/PVA scaffolds on days 4,7 , and $11(P<0.05$; Figure $5 \mathrm{~A})$, indicating that the incorporation of PRF enhanced BMSC proliferation. The cell proliferation rate on the nonprinted $\mathrm{BCP} / \mathrm{PVA}$ and BCP/PVA/PRF scaffolds slowed at day 11, which may be due to contact inhibition as a result of the cells covering the limited surface area. This suggestion is consistent with the SEM analyses (Figure 5C), in which the nonprinted scaffolds were observed to be overgrown with BMSCs, while the printed scaffolds still possessed sufficient surface area for cell proliferation. These results suggest that the incorporation of PRF enhanced early BMSC proliferation, while the printed scaffolds provide a spatially large interconnected structure for cell adhesion and proliferation.

\section{Osteogenic differentiation of BMSCs}

The ability of BMSCs to differentiate toward the osteogenic lineage was evaluated by assessing the ALP activity of the cells seeded on the scaffolds. ALP is considered as an early marker of osteogenic differentiation; ${ }^{47}$ ALP activity represents the activity of a single intracellular enzyme. On both days 7 and 14 after seeding, BMSCs on the nonprinted BCP/ PVA/PRF scaffolds had higher ALP activity than cells on the nonprinted $\mathrm{BCP} / \mathrm{PVA}$ scaffolds $(P<0.05)$ and BMSCs on the printed $\mathrm{BCP} / \mathrm{PVA} / \mathrm{PRF}$ scaffolds had higher ALP activity than cells on the printed BCP/PVA scaffolds $(P<0.05$; 


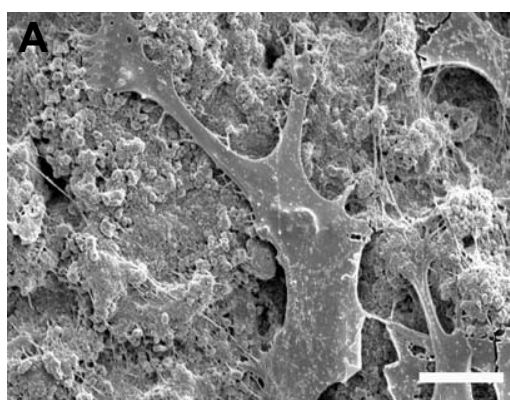

D
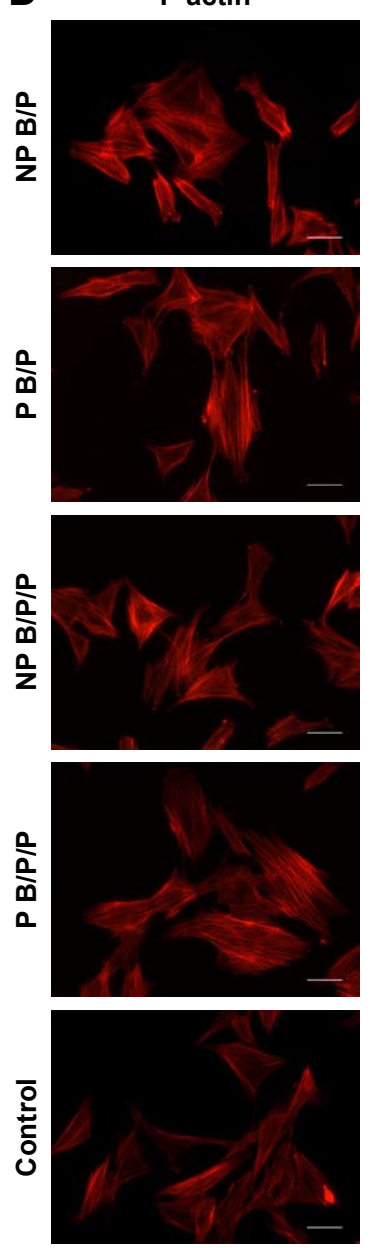
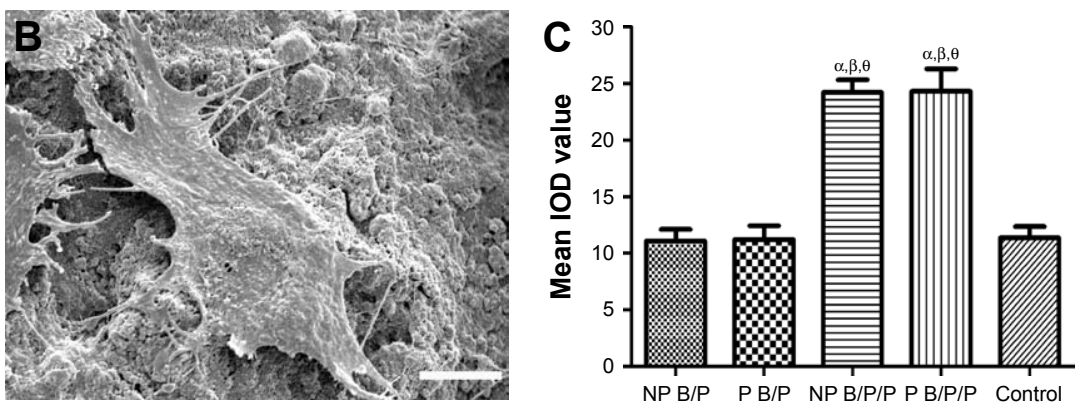

Vinculin
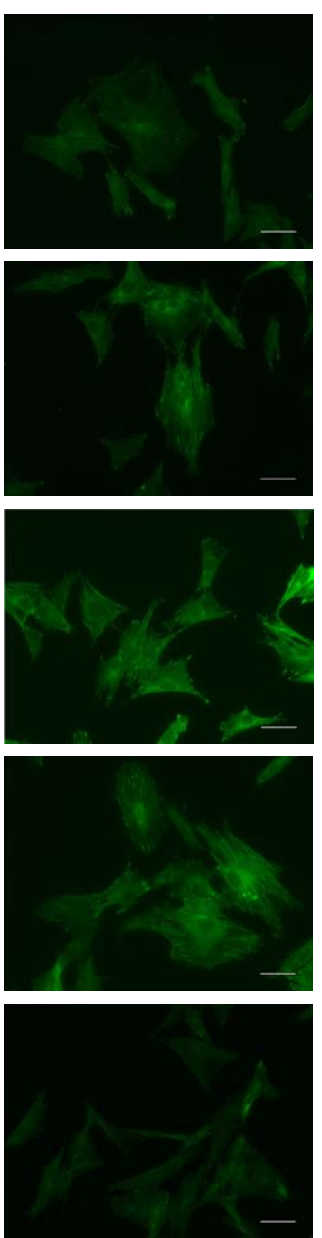

DAPI
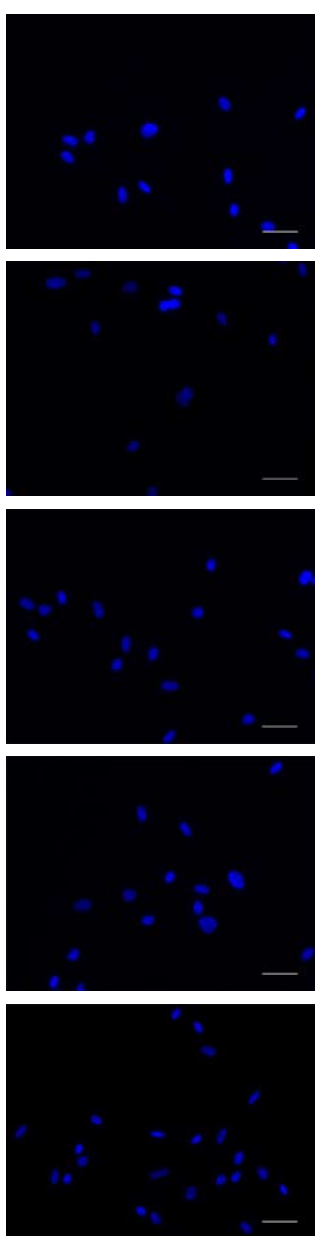
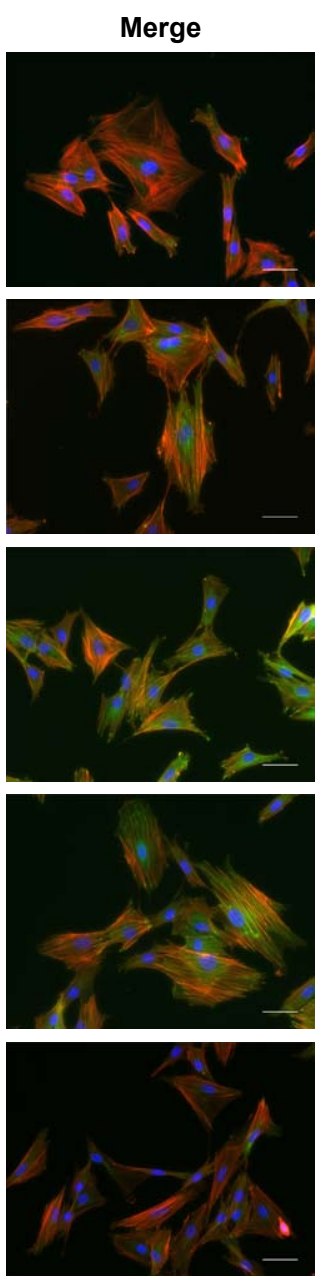

Figure 4 Assessment of BMSC adhesion to the printed scaffolds.

Notes: Scanning electron microscopy analysis of the adhesion status of BMSCs on P B/P scaffolds $(\mathbf{A})$ and $\mathrm{P}$ B/P/P scaffolds (B) at $24 \mathrm{~h}$ after seeding; scale bars: $10 \mu \mathrm{m}$. (C) Quantitative analysis of the integral optical density value for vinculin fluorescence for BMSCs seeded on NP B/P scaffolds, P B/P scaffolds, NP B/P/P scaffolds, and P B/P/P scaffolds. Results are mean \pm standard deviation $(\chi \pm \mathrm{SD}), \mathrm{n}=3$; ${ }^{\alpha} P<0.05$ vs NP B/P scaffolds; ${ }^{\beta} P<0.05$ vs $P$ B/P scaffolds; ${ }^{\beta} P<0.05$ vs cell culture plates. (D) Representative images of immunofluorescence staining for F-actin (red), vinculin (green), and nuclei (4',6-diamidino-2-phenylindole, blue) in BMSCs seeded on scaffolds or cell culture plates (control) at $24 \mathrm{~h}$ after seeding; scale bars: $50 \mu \mathrm{m}$.

Abbreviations: BMSCs, bone marrow-derived mesenchymal stem cells; IOD, integral optical density; NP B/P, nonprinted biphasic calcium phosphate/polyvinyl alcohol; NP B/P/P, nonprinted biphasic calcium phosphate/polyvinyl alcohol/platelet-rich fibrin; P B/P, printed biphasic calcium phosphate/polyvinyl alcohol; P B/P/P, printed biphasic calcium phosphate/polyvinyl alcohol/platelet-rich fibrin.

Figure 5B), although ALP activity was lower on day 14 than on day 7. These results are similar to those of Man et al ${ }^{48}$ and confirm that the incorporation of PRF enhanced the ability of the scaffolds to promote osteogenic differentiation.

Assessment of relative mRNA expression levels by qRTPCR (Figure 6) revealed that BMSCs cultured on the printed
BCP/PVA/PRF scaffolds expressed significantly higher levels of Alp, Col1A1, Opn, and Runx-2 on days 7 and 14 compared with BMSCs on the other scaffolds (all $P<0.05$ ). These results indicate that the incorporation of $\mathrm{PRF}$ and the $3 \mathrm{D}$ structure of the printed BCP/PVA/PRF scaffolds simulated the ability of the bone microenvironment to promote BMSC differentiation. 

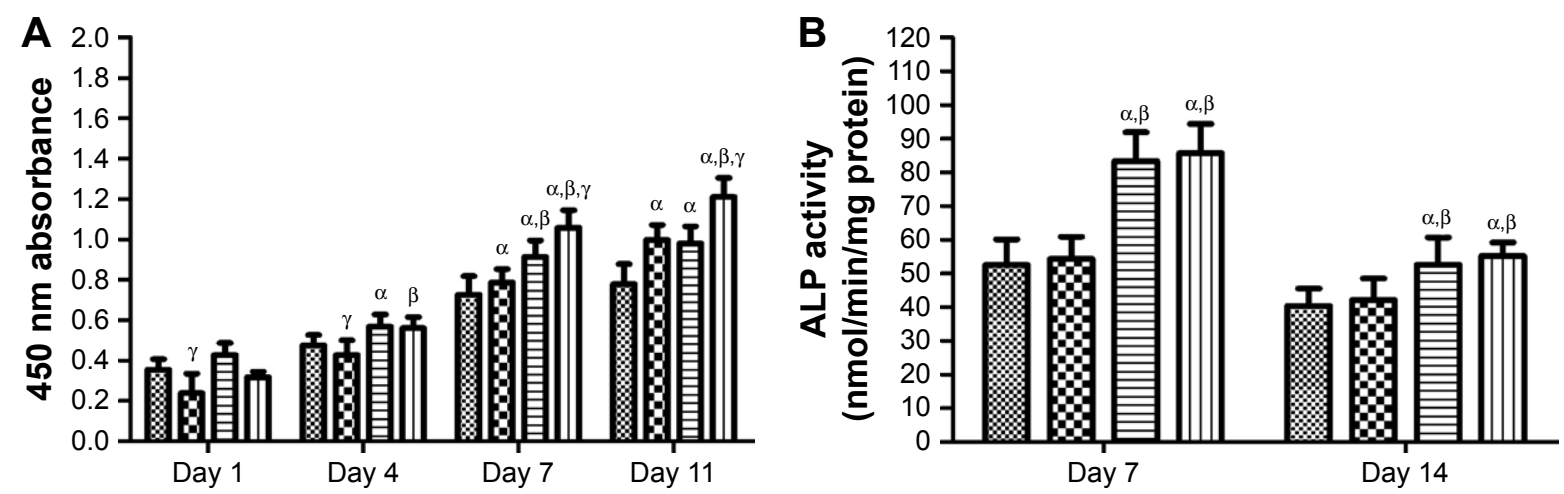

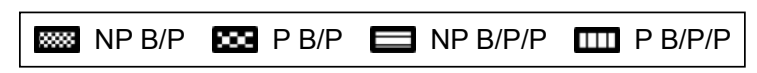

C NP B/P
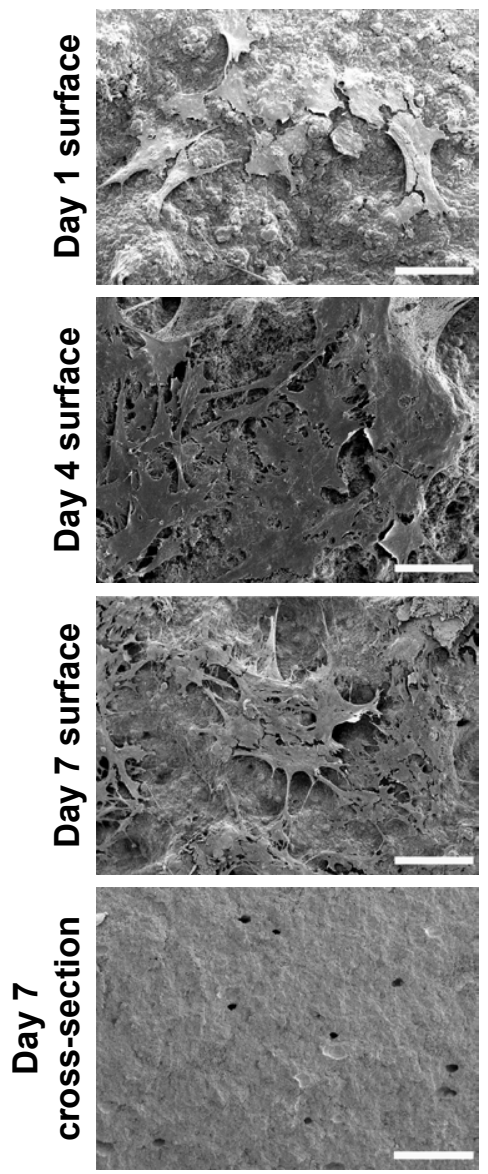

P B/P
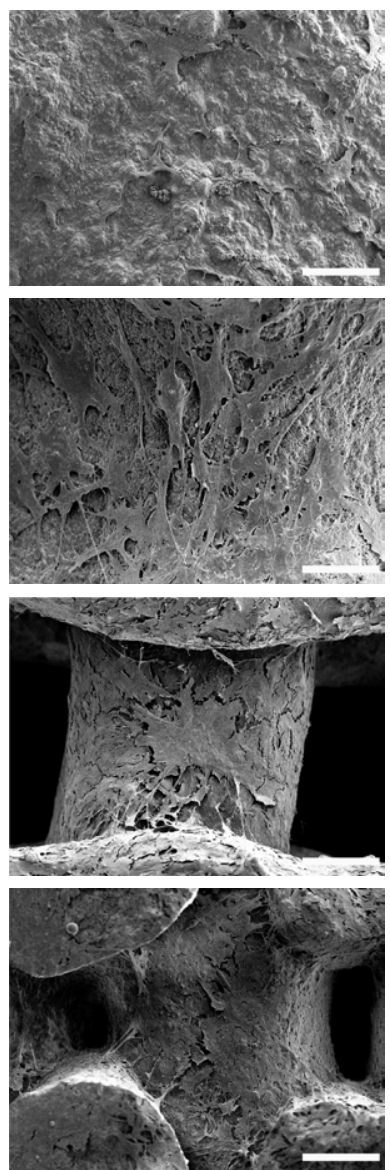

NP B/P/P
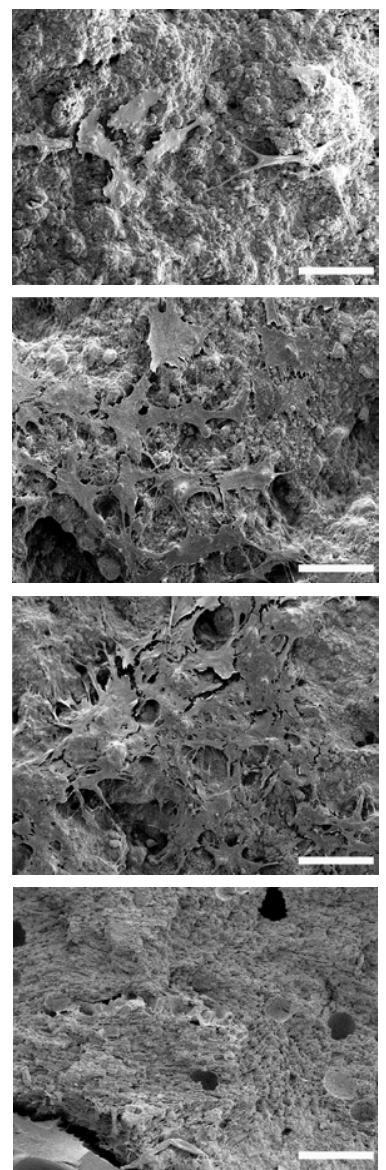

Day 14 

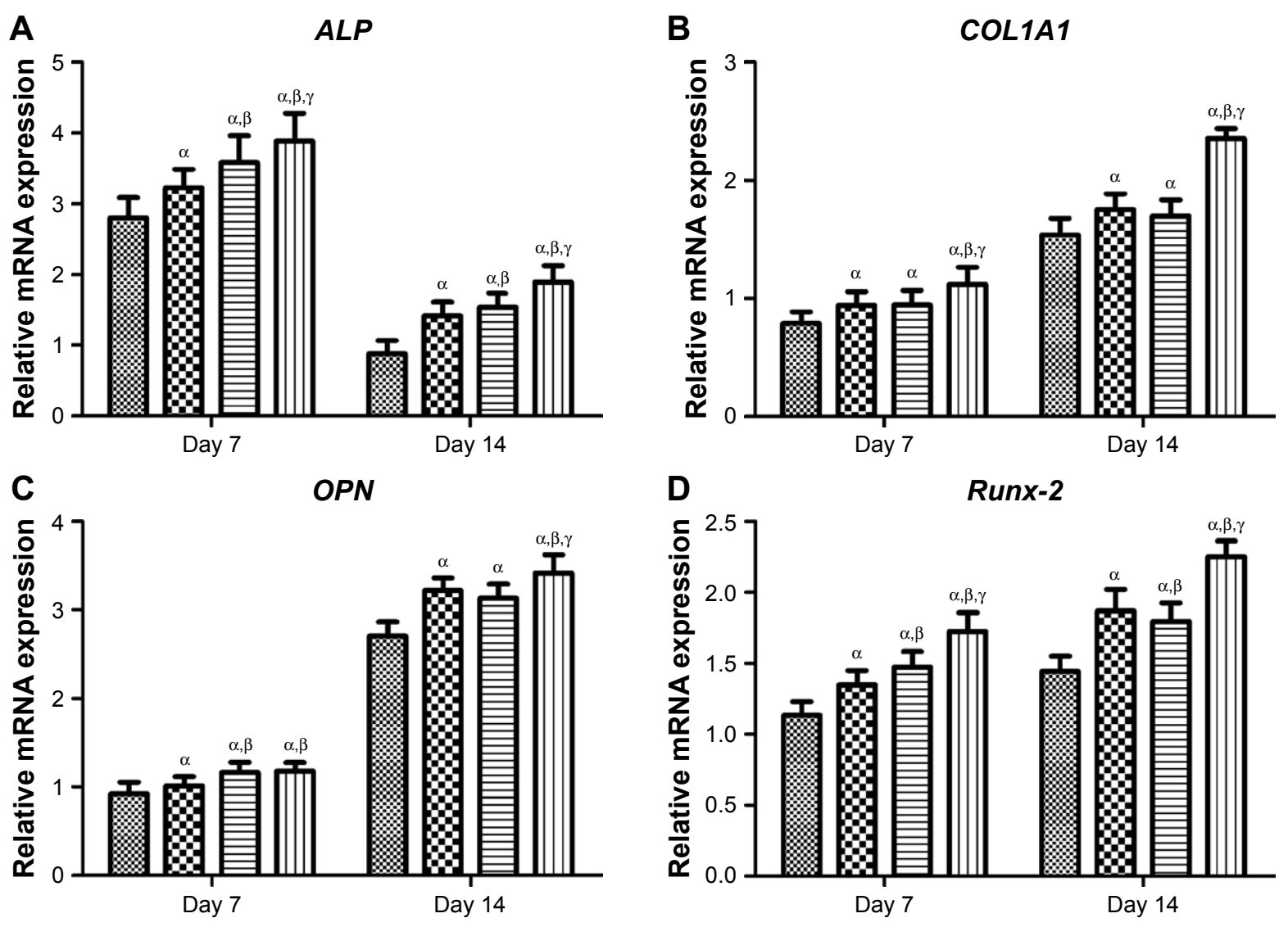

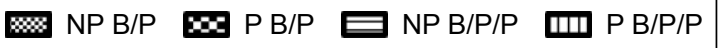

Figure 6 Quantitative real-time polymerase chain reaction analysis of gene expression in BMSCs at 7 and 14 days after seeding onto scaffolds.

Notes: BMSCs were seeded onto NP B/P scaffolds, P B/P scaffolds, NP B/P/P scaffolds, or P B/P/P scaffolds. (A) Alp, (B) CollA I, (C) Opn, and (D) Runx-2. Results are mean \pm standard deviation $(\chi \pm S D), n=3 ;{ }^{\alpha} P<0.05$ vs NP B/P scaffolds; ${ }^{\beta} P<0.05$ vs $P$ B/P scaffolds; $\gamma P<0.05$ vs NP B/P/P scaffolds.

Abbreviations: BMSCs, bone marrow-derived mesenchymal stem cells; NP B/P, nonprinted biphasic calcium phosphate/polyvinyl alcohol; NP B/P/P, nonprinted biphasic calcium phosphate/polyvinyl alcohol/platelet-rich fibrin; P B/P, printed biphasic calcium phosphate/polyvinyl alcohol; P B/P/P, printed biphasic calcium phosphate/polyvinyl alcohol/platelet-rich fibrin.

In agreement with the micro-CT analysis, all scaffold groups had higher BV/TV values than the control group $(P<0.05)$. However, the nonprinted and printed BCP/ PVA/PRF scaffolds had higher BV/TV values than the nonprinted and printed BCP/PVA scaffolds, respectively, at 8 and 12 weeks $(P<0.05)$, indicating that the incorporation of PRF increased new bone formation. The printed BCP/ PVA/PRF scaffolds exhibited the highest bone volume formation compared with the other scaffolds $(P<0.05)$ (Figure 7B).

RSV/SV analysis showed that the printed BCP/PVA/PRF scaffolds had a significantly higher degradation rate than the nonprinted BCP/PVA scaffolds at 4 weeks $(P<0.05)$. At 8 weeks, the printed BCP/PVA scaffolds had a higher degradation rate than the nonprinted $\mathrm{BCP} / \mathrm{PVA}$ scaffolds (78.73 \pm 2.45 vs $86.27 \pm 2.53 ; P=0.0110)$ and the printed $\mathrm{BCP} / \mathrm{PVA} / \mathrm{PRF}$ scaffolds had a higher degradation rate than the nonprinted BCP/PVA/PRF scaffolds $(74.16 \pm 2.27$ vs $83.07 \pm 2.56 ; P=0.0108)$. Similar trends were observed at 12 weeks, when the printed BCP/PVA scaffolds had a lower RSV/SV value than the nonprinted BCP/PVA scaffolds (70.49 \pm 2.82 vs $79.36 \pm 2.31 ; P=0.0136)$ and the printed $\mathrm{BCP} / \mathrm{PVA} / \mathrm{PRF}$ scaffolds had a lower RSV/SV value than the nonprinted $\mathrm{BCP} / \mathrm{PVA} / \mathrm{PRF}$ scaffolds $(65.07 \pm 2.66 \mathrm{vs}$ 77.75 $\pm 2.74 ; P=0.0045$ ) (Figure 7C).

\section{Histological staining}

Images of fluorescent double staining for tetracycline (yellow) and calcein (green) are shown in Figure 8A-E. The distance between the yellow and green fluorescent bands was higher on the nonprinted BCP/PVA/PRF scaffolds than the nonprinted BCP/PVA scaffolds $(28.65 \pm 2.13$ vs $20.47 \pm 1.97 \mu \mathrm{m} ; P<0.05)$. Moreover, the distance between the fluorescent bands was almost 1.4-fold higher on the printed BCP/PVA/PRF scaffolds than the printed BCP/PVA scaffolds (29.87 \pm 3.25 vs $20.64 \pm 2.82 \mu \mathrm{m} ; P<0.05)$. 
A
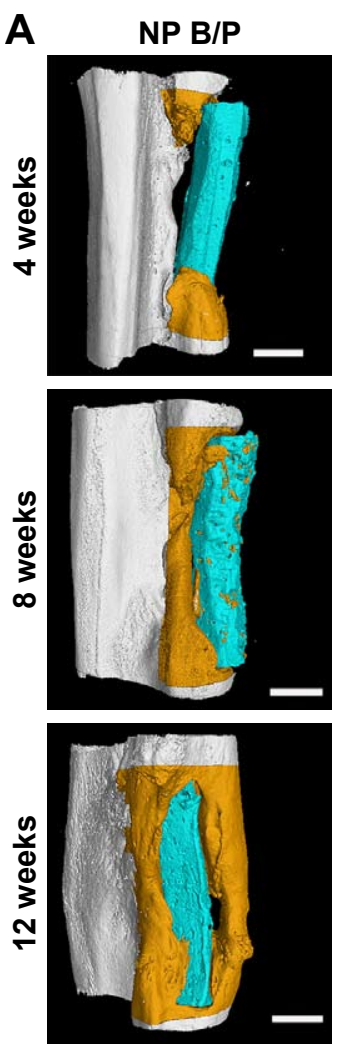

B

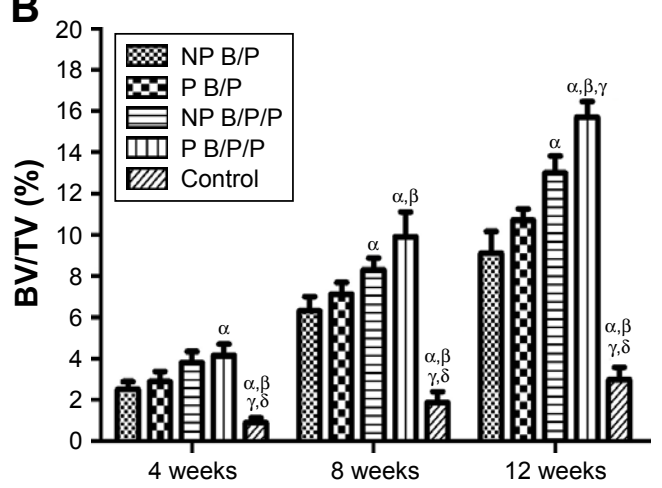

P B/P
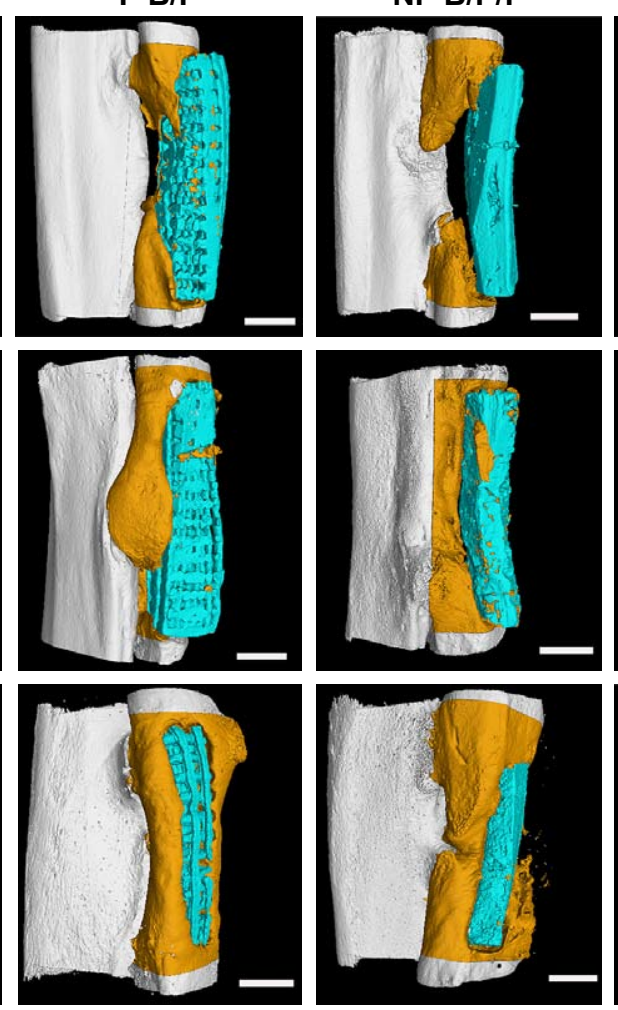

C
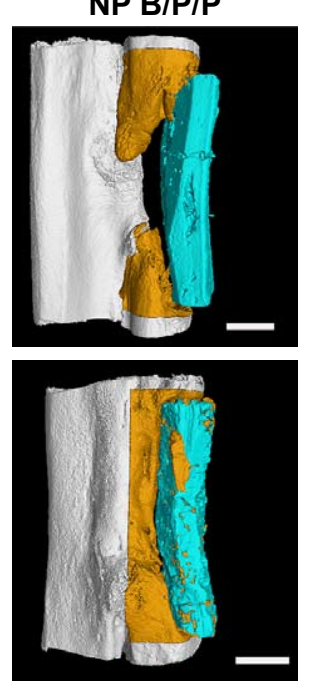

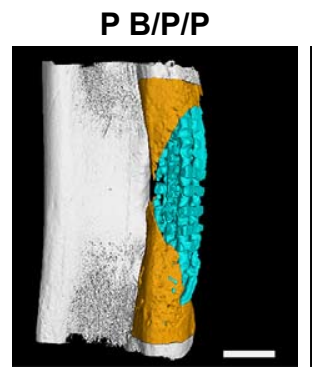

Control
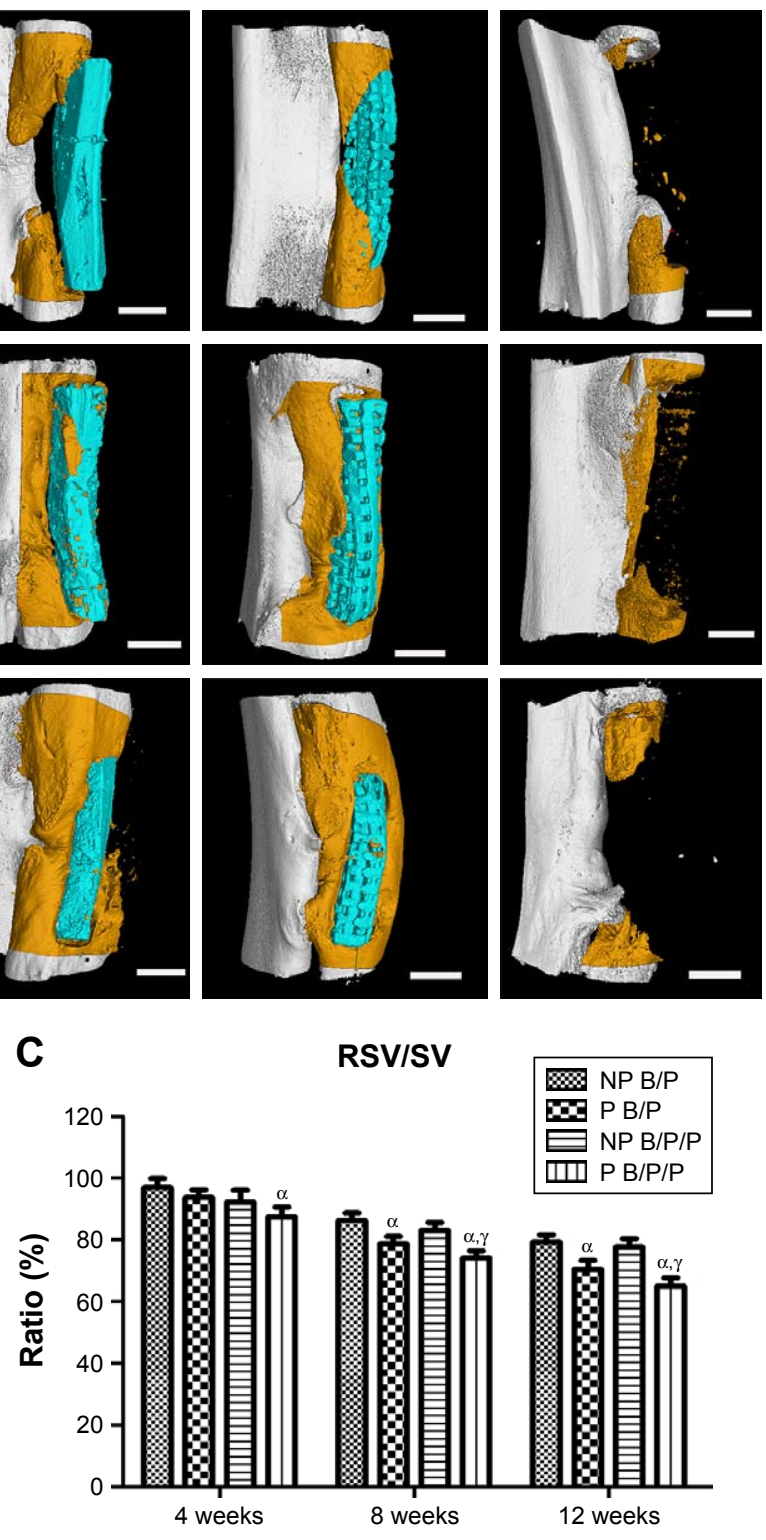

RSVISV

12 weeks

Figure 7 Micro-CT imaging and osteogenic analysis.

Notes: (A) Micro-CT scans and 3D reconstructions to visualize healing of critical size bone defects in the rabbit radius at 4, 8 , and 12 weeks after implantation of scaffolds seeded with BMSCs. Defects were implanted with NP B/P scaffolds, P B/P scaffolds, NP B/P/P scaffolds, P B/P/P scaffolds, or no scaffold (control). The scaffolds are shown in blue, and newly formed radius bone is shown in dark orange in the micro-CT images; scale bars: $3 \mathrm{~mm}$. (B) Bone volume fraction. (C) RSV/SV values. Results are

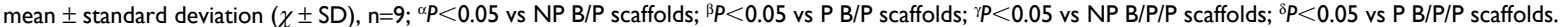

Abbreviations: BMSCs, bone marrow-derived mesenchymal stem cells; BV, bone volume; 3D, three-dimensional; NP B/P, nonprinted biphasic calcium phosphate/polyvinyl alcohol; NP B/P/P, nonprinted biphasic calcium phosphate/polyvinyl alcohol/platelet-rich fibrin; micro-CT, micro-computed tomography; P B/P, printed biphasic calcium phosphate/polyvinyl alcohol; P B/P/P, printed biphasic calcium phosphate/polyvinyl alcohol/platelet-rich fibrin; RSV, residual scaffold volume; SV, scaffold volume.

The MARs calculated from the tetracycline and calcein fluorescent double staining were higher for all four groups of scaffolds than for the control group without a scaffold $(P<0.05$; Figure $8 \mathrm{~F})$. The MARs of the nonprinted and printed $\mathrm{BCP} / \mathrm{PVA} / \mathrm{PRF}$ scaffolds were higher than those of the nonprinted and printed $\mathrm{BCP} / \mathrm{PVA}$ scaffolds, respectively (both $P<0.05$ ).

To evaluate the tissue response to the implanted scaffolds and assess the defect healing progress, we performed histological analysis using Van Gieson's staining at 4, 8, and 12 weeks (Figure 9). By 4 weeks after implantation, bone callus (red) was prominent in the specimens implanted with the nonprinted and printed BCP/PVA/PRF scaffolds. Moreover, large areas of newly formed callus were observed to extend into the regular pores of the printed BCP/PVA/ PRF scaffolds. In comparison, numerous stromal cells and fibrous tissue (blue) surrounded the nonprinted and printed BCP/PVA scaffolds. 

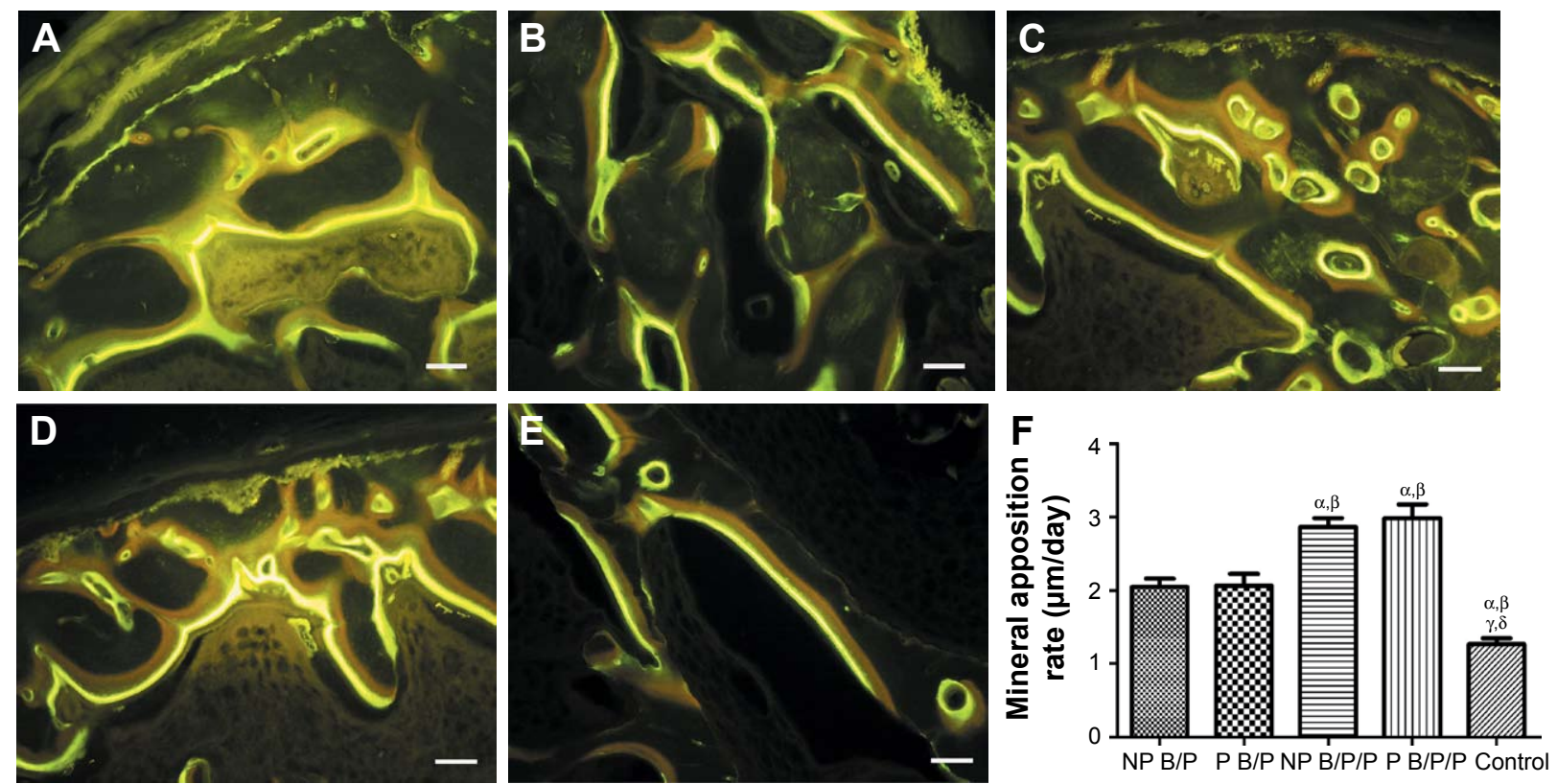

Figure 8 Mineral apposition rates.

Notes: Images of fluorescence double staining for tetracycline (yellow) and calcein (green) in the defects implanted with (A) NP B/P scaffolds, (B) P B/P scaffolds, (C) NP B/P/P scaffolds, (D) P B/P/P scaffolds (E), or control group without implanted scaffolds; scale bars: $100 \mu \mathrm{m}$. (F) Mineral apposition rates for NP B/P scaffolds, P B/P scaffolds, NP B/P/P scaffolds, and $P B / P / P$ scaffolds. Results are mean \pm standard deviation $(\chi \pm S D), n=3 ;{ }^{\alpha} P<0.05$ vs NP B/P scaffolds; ${ }^{\beta} P<0.05$ vs $P B / P$ scaffolds; ${ }^{\gamma} P<0.05$ vs NP $B / P / P$ scaffolds; ${ }^{\delta} P<0.05$ vs $P B / P / P$ scaffolds.

Abbreviations: NP B/P, nonprinted biphasic calcium phosphate/polyvinyl alcohol; NP B/P/P, nonprinted biphasic calcium phosphate/polyvinyl alcohol/platelet-rich fibrin; P B/P, printed biphasic calcium phosphate/polyvinyl alcohol; P B/P/P, printed biphasic calcium phosphate/polyvinyl alcohol/platelet-rich fibrin.

At 8 weeks after implantation, the formation of new bone had progressed markedly in the nonprinted and printed $\mathrm{BCP} /$ PVA/PRF scaffold groups. Sponge-like bone tissues were observed in both the peripheral and central zones of the printed
BCP/PVA/PRF scaffolds. Furthermore, due to the ingrowth of new bone, the edges of the printed BCP/PVA/PRF scaffolds began to degrade and fracture. In contrast, only cartilage tissues enveloped the nonprinted and printed BCP/PVA scaffolds.
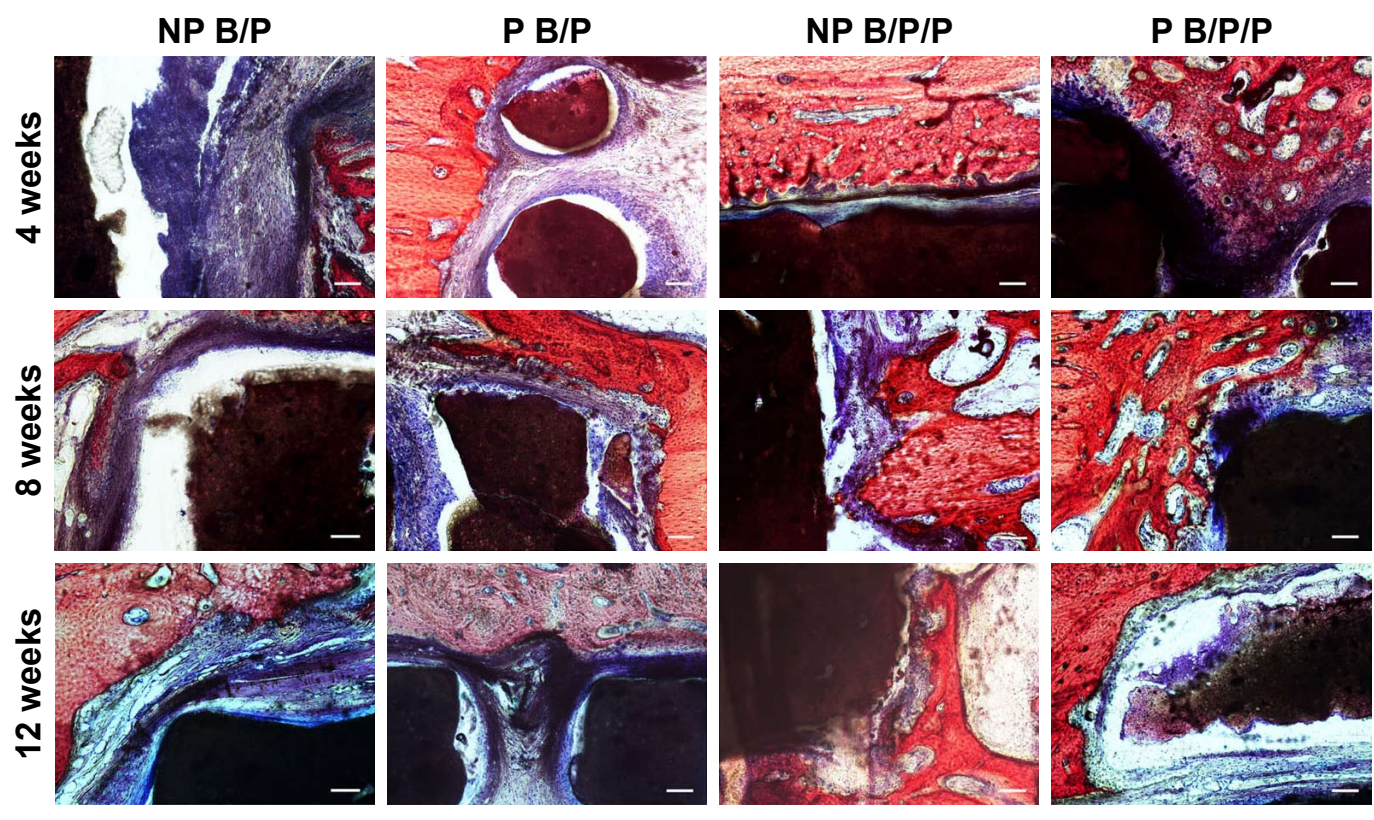

Figure 9 Histological staining of new bone formation after implantation of scaffolds seeded with BMSCs into critical size bone defects in the rabbit radius. Notes: The newly formed bone is stained red, fibrous tissue is blue and the scaffolds are black (Van Gieson's staining); scale bars: $100 \mu \mathrm{m}$.

Abbreviations: BMSCs, bone marrow-derived mesenchymal stem cells; NP B/P, nonprinted biphasic calcium phosphate/polyvinyl alcohol; NP B/P/P, nonprinted biphasic calcium phosphate/polyvinyl alcohol/platelet-rich fibrin; P B/P, printed biphasic calcium phosphate/polyvinyl alcohol; P B/P/P, printed biphasic calcium phosphate/polyvinyl alcohol/platelet-rich fibrin. 
At 12 weeks after implantation, woven bone combined with lamellar bone was observed around the residual scaffolds in the nonprinted and printed $\mathrm{BCP} / \mathrm{PVA} / \mathrm{PRF}$ groups and significant degradation of the printed BCP/PVA/PRF scaffolds had occurred. In contrast, although a small quantity of lamellar bone was present in the areas close to the host bone around the nonprinted and printed BCP/PVA scaffolds, this bone was thin and irregular.

\section{Discussion}

The 3D printing of bone scaffolds is a promising approach as it combines the precision of $3 \mathrm{D}$ printing techniques with tissue engineering to fabricate bionic bone implants with a desired shape and internal structure. ${ }^{49}$

The ideal bone tissue engineering scaffold should possess a number of characteristics. First, the raw materials used to fabricate the scaffolds should be easy to acquire and simple to prepare. ${ }^{49}$ In this article, we demonstrate the feasibility and simplicity of fabricating $3 \mathrm{D}$ printed $\mathrm{BCP} / \mathrm{PVA} / \mathrm{PRF}$ scaffolds under mild and cytocompatible conditions without the use of unnecessary harmful substances. The printing process was performed at a low temperature and minimally affected the biological activity of PRF. This is the first BCP/PVA/ PRF scaffold to be printed at a low temperature and retain the biological activity of the incorporated factors. In contrast, the printed scaffolds described by $\mathrm{He}$ et $\mathrm{al}^{50}$ needed to be incubated for $30 \mathrm{~min}$ at $150^{\circ} \mathrm{C}$ in a muffle furnace to facilitate cross-linking, which would destroy the biological activity of the incorporated growth factors.

Second, the mechanical properties of the ideal scaffold need to be the optimal combination of flexibility and rigidity. ${ }^{51}$ As described in our previous article, ${ }^{19}$ we can fabricate large scaffolds (for the tibial plateau model) up to $3 \mathrm{~cm}$ in length using a FFP printer. The production of grafts with desired shapes and internal structures to repair critical-size defects in the clinic remains a challenge. ${ }^{52}$ Robocasting, as employed in this study, may be a possible method for generating large implants for clinical use as the resulting scaffolds can fully support their own weight during assembly. ${ }^{51}$

Third, porosity and pore size need to be sufficiently large to allow the formation and ingrowth of new bone tissue. ${ }^{49}$ Integrative SEM revealed that the pore size of the printed $\mathrm{BCP} / \mathrm{PVA} / \mathrm{PRF}$ scaffold is $\sim 500-600$ and $>300 \mu \mathrm{m}$ is favorable for vascularization. ${ }^{53}$ The porosity of the printed $\mathrm{BCP} /$ PVA/PRF scaffolds reached up to 50\%. While the compressive modulus of the printed BCP/PVA/PRF scaffolds was lower than that of the corresponding nonprinted BCP/PVA/ PRF scaffolds, it was higher than our previously reported values for printed HAp/collagen scaffolds. ${ }^{19}$ In the field of biomaterials, there is always a compromise between porosity and mechanical properties. ${ }^{54}$ High porosity is necessary for oxygen and nutrient transportation, new tissue formation, vascularization, and neurotization. However, bionic bone needs to be stiff enough to provide support for cell attachment and bear the forces conducted from the adjacent bone tissues. Although the mean compressive modulus of the printed $\mathrm{BCP} / \mathrm{PVA} / \mathrm{PRF}$ was lower than that of cancellous bone $(\sim 2-20 \mathrm{mPa}),{ }^{55}$ printed BCP/PVA/PRF could be applied in combination with orthopedic surgical internal fixation to promote biological osteosynthesis. ${ }^{49}$

Finally, in terms of ideal properties, a scaffold should have favorable biocompatibility and biological activity and its microenvironment should facilitate mineralization and vascularization..$^{49}$ With respect to biocompatibility, printed scaffolds must facilitate the survival, migration, proliferation, and differentiation of seeded or recruited cells. ${ }^{56}$ The regeneration of bone tissue occurs via complicated temporal and spatial coordination of a massive range of processes involving different cells that are induced by a vast number of signals from the ECM. ${ }^{4}$ However, synthetic biomaterials lack the necessary signals and surface modifications to promote efficient cell attachment and migration, let alone recapitulate the bone regeneration processes. ${ }^{4,49} \mathrm{PRF}$ has the $3 \mathrm{D}$ organization of a fibrin network, which promotes cytokine enmeshment and facilitates cellular migration. PRF granules were distributed homogeneously throughout the low temperature printed scaffolds generated in this study and were also found to be distributed on the scaffold surface, which increased surface roughness and provided anchorage sites for cell adhesion. Moreover, the introduction of PRF significantly improved the hydrophilicity and surface roughness of the scaffolds, which improved the seeding efficiency; this is consistent with other research, showing that the introduction of peptides onto polymer surfaces promoted cell adhesion. ${ }^{57}$

In addition to enhance cell adhesion, the incorporation of PRF also promoted BMSC proliferation on the scaffolds, as indicated by toxicity and cell proliferation assays and SEM analysis at various time points, confirming that PRF provides suitable sites for cell attachment. Immunofluorescent staining and quantitative assessment suggested that BMSCs seeded on the scaffolds containing PRF expressed high levels of vinculin, confirming that the printed BCP/PVA/PRF scaffolds provide a favorable microenvironment for the adhesion, extension, and migration of BMSCs.

Cell proliferation assays and SEM analysis confirmed that BMSCs proliferated well on each of the four types of scaffolds tested. By day 7 , the surfaces of all scaffolds were covered with cells, especially the printed BCP/PVA/PRF 
scaffolds. The interiors of the printed scaffolds were also covered by cells. Higher numbers of cells were detected on the printed BCP/PVA and BCP/PVA/PRF scaffolds than on the corresponding nonprinted scaffolds, as the higher specific surface area of the printed scaffolds supported the growth of more cells. ${ }^{58}$ The ELISA results confirmed the slow, sustained release of growth factors from the printed $\mathrm{BCP} /$ PVA/PRF scaffolds, which is consistent with the findings of Zhao et $\mathrm{al}^{27}$ and Jayasree et al. ${ }^{45}$ These researchers used pure PRF or PRF loaded into gelatine-nanoHAp fibrous scaffolds. VEGF and PDGF mainly promote angiogenesis through recruiting endothelial cells and forming tubules. ${ }^{59}$ PDGF also promotes BMSC recruitment, ${ }^{60}$ and IGF plays a part in neoangiogenesis. ${ }^{61}$ TGF mainly recruits osteoblast precursors. ${ }^{62}$ While some other researchers have tried to release single growth factors from scaffolds, the process of bone regeneration requires multiple growth factors working together to promote the activity of osteogenic-related cells. ${ }^{63,64}$ The prolonged release of growth factors from PRF may provide proliferation and differentiation stimuli for BMSCs to promote bone formation.

One major factor that limits the application of criticalsize tissue engineered bone replacements in the clinic is an insufficient supply of nutrients and oxygen throughout the entire scaffold. ${ }^{49,65}$ Observations of cell growth, proliferation, and adherent morphology indicated that the BMSCs on the internal surfaces of the printed scaffolds could obtain oxygen and nutrients and efficiently transport metabolic waste.

The cells seeded onto or recruited to tissue engineered scaffolds must differentiate to enable complete repair. ${ }^{66}$ ALP is an early marker of BMSC differentiation toward osteoblasts. ${ }^{67}$ BMSCs on the printed BCP/PVA/PRF scaffolds exhibited higher ALP activity than cells on the printed $\mathrm{BCP} / \mathrm{PVA}$ scaffolds, indicating that the incorporation of PRF promoted more rapid differentiation toward the osteoblast lineage. These results are similar to those of Man et al, ${ }^{48}$ who showed that ALP activity was significantly elevated at day 7 in PRP-laden scaffolds compared with non-PRPladen scaffolds. The qRT-PCR analysis confirmed that the printed BCP/PVA/PRF scaffolds promoted osteogenic differentiation. In fact, these effects could be attributed to the combination of a high surface area, a 3D stereoscopic culture area, ${ }^{68}$ and the presence of PRF in the printed BCP/ PVA/PRF scaffolds. ${ }^{48,69}$

To further explore the osteoinductivity and osteoconductivity of the printed BCP/PVA/PRF scaffolds in vivo, the cell/scaffold constructs were implanted into critical-size bone defects in the rabbit radius. Micro-CT imaging and the $\mathrm{BV} / \mathrm{TV}$ ratio revealed that the printed $\mathrm{BCP} / \mathrm{PVA} / \mathrm{PRF}$ scaffolds enhanced regeneration of the bone defects and accelerated defect healing compared with the other scaffolds tested. Micro-CT revealed that some newly formed bone had penetrated into the pores of the printed BCP/PVA/PRF scaffolds; Van Gieson's staining confirmed this observation and indicated that the newly formed bone surrounding the printed BCP/PVA/PRF scaffolds was firmly attached. Furthermore, inflammatory cell infiltration was not detected at the bone/ scaffold interface, indicating the absence of a foreign body reaction and confirming the good biocompatibility of all four scaffolds tested.

Ideally, a scaffold should degrade over time and eventually be replaced by newly formed bone. ${ }^{70}$ The incorporation of PRF accelerated the formation of new bone and scaffold degradation. The RSV/SV values demonstrated that the printed scaffolds degraded faster than the nonprinted scaffolds, which might be due to their higher contact area with tissue and the fact mononuclear macrophages could enter the pores of the printed scaffolds. The degradation rate is similar to that of BCP scaffolds fabricated by He et al. ${ }^{71}$ Overall, the in vivo studies demonstrated that the implantation of all low temperature printed scaffolds, especially the printed $\mathrm{BCP} /$ PVA/PRF scaffolds, improved the repair of critical-size bone defects.

The main deficiency of the printed BCP/PVA/PRF scaffold is its low mechanical strength and slow degradation rate; however, from the perspective of an orthopedic surgeon, these scaffolds could be applied in combination with orthopedic surgical internal fixation to obtain biological osteosynthesis. The mechanical strength and degradation rate of the printed $\mathrm{BCP} / \mathrm{PVA} / \mathrm{PRF}$ scaffold needs to be optimized. In future research, the degradation rate of the scaffolds could be optimized by adding other biomaterials that degrade more rapidly.

\section{Conclusion}

We demonstrated the feasibility of preparing 3D printed BCP/PVA/PRF scaffolds using a simple, low-temperature method. We found that the resulting scaffolds exhibited good biological activity and biocompatibility in vitro and in vivo and promoted appropriate new bone formation in vivo. The biological activity of PRF was retained during the $3 \mathrm{D}$ printing process, and the presence of PRF in the biocompatible microenvironment of the scaffold provided cell binding sites and promoted the adhesion, proliferation, and differentiation of BMSCs. In addition, the prolonged release of growth factors from PRF may provide proliferation and 
differentiation stimuli. When the cell/scaffold constructs were transplanted into critical-size bone defects in the rabbit radius, the presence of PRF appeared to provide osteoconductive and osteoinductive stimuli that promoted appropriate bone regeneration and repair.

\section{Acknowledgments}

Dr Qingfeng Zeng and Mr Xin Zhang from Xi'an Particle Cloud Biotechnology are acknowledged for providing instrument facilities and knowledge of material science. This study was funded by the State Key Project of Research and Development of China, grant number: 2016YFC1100300, and the National Natural Science Foundation of China, grant numbers: 81672189 and 81430049 . Yue Song, Kaifeng Lin, and Shu He are joint first authors.

\section{Disclosure}

The authors report no conflicts of interest in this work.

\section{References}

1. Petite H, Viateau V, Bensaïd W, et al. Tissue-engineered bone regeneration. Nat Biotechnol. 2000;18(9):959-963.

2. Tampieri A, Sprio S, Sandri M, Valentini F. Mimicking natural bio-mineralization processes: a new tool for osteochondral scaffold development. Trends Biotechnol. 2011;29(10):526-535.

3. Langer R, Tirrell DA. Designing materials for biology and medicine. Nature. 2004;428(6982):487-492.

4. Lutolf MP, Hubbell JA. Synthetic biomaterials as instructive extracellular microenvironments for morphogenesis in tissue engineering. Nat Biotechnol. 2005;23(1):47-55.

5. Murphy SV, Atala A. 3D bioprinting of tissues and organs. Nat Biotechnol. 2014;32(8):773-785.

6. Song K, Li L, Yan X, et al. Fabrication and development of artificial osteochondral constructs based on cancellous bone/hydrogel hybrid scaffold. J Mater Sci Mater Med. 2016;27(6):114.

7. Kane R, Mal PX. Mimicking the nanostructure of bone matrix to regenerate bone. Mater Today (Kidlington). 2013;16(11):418-423.

8. Dadhich P, Das B, Pal P, et al. A simple approach for an eggshell-based 3D-printed osteoinductive multiphasic calcium phosphate scaffold. ACS Appl Mater Interfaces. 2016;8(19):11910-11924.

9. Fujita T, Saito N, Minakata K, Imai M, Yamazaki K, Kimura T. Transfemoral transcatheter aortic valve implantation in the presence of a mechanical mitral valve prosthesis using a dedicated TAVI guidewire: utility of a patient-specific three-dimensional heart model. Cardiovasc Interv Ther. 2016;32(3):308-311.

10. You W, Liu LJ, Chen HX, et al. Application of 3D printing technology on the treatment of complex proximal humeral fractures (Neer3-part and 4-part) in old people. Orthop Traumatol Surg Res. 2016;102(7): 897-903.

11. Wang MO, Vorwald CE, Dreher ML, et al. Evaluating 3D-printed biomaterials as scaffolds for vascularized bone tissue engineering. Adv Mater. 2015;27(1):138-144.

12. Zhu W, Yan C, Shi Y, et al. A novel method based on selective laser sintering for preparing high-performance carbon fibres/polyamide12/ epoxy ternary composites. Sci Rep. 2016;6:33780.

13. Alhijjaj M, Belton P, Qi S. An investigation into the use of polymer blends to improve the printability of and regulate drug release from pharmaceutical solid dispersions prepared via fused deposition modeling (FDM) 3D printing. Eur J Pharm Biopharm. 2016;108:111-125.
14. Ishida Y, Miyasaka T. Dimensional accuracy of dental casting patterns created by 3D printers. Dent Mater J. 2016;35(2):250-256.

15. van Bochove B, Hannink G, Buma P, Grijpma DW. Preparation of designed poly(trimethylene carbonate) meniscus implants by stereolithography: challenges in stereolithography. Macromol Biosci. 2016; 16(12):1853-1863.

16. Barui S, Chatterjee S, Mandal S, Kumar A, Basu B. Microstructure and compression properties of 3D powder printed Ti-6Al-4V scaffolds with designed porosity: experimental and computational analysis. Mater Sci Eng C Mater Biol Appl. 2017;70(pt 1):812-823.

17. Loh QL, Choong C. Three-dimensional scaffolds for tissue engineering applications: role of porosity and pore size. Tissue Eng Part B Rev. 2013;19(6):485-502.

18. Miranda P, Saiz E, Gryn K, Tomsia AP. Sintering and robocasting of beta-tricalcium phosphate scaffolds for orthopaedic applications. Acta Biomater. 2006;2(4):457-466.

19. Lin KF, He S, Song Y, et al. Low-temperature additive manufacturing of biomimic three-dimensional hydroxyapatite/collagen scaffolds for bone regeneration. ACS Appl Mater Interfaces. 2016;8(11):6905-6916.

20. Palmer LC, Newcomb CJ, Kaltz SR, Spoerke ED, Stupp SI. Biomimetic systems for hydroxyapatite mineralization inspired by bone and enamel. Chem Rev. 2008;108(11):4754-4783.

21. Baino F, Novajra G, Vitale-Brovarone C. Bioceramics and scaffolds: a winning combination for tissue engineering. Front Bioeng Biotechnol. 2015;3:202.

22. Fellah BH, Gauthier O, Weiss P, Chappard D, Layrolle P. Osteogenicity of biphasic calcium phosphate ceramics and bone autograft in a goat model. Biomaterials. 2008;29(9):1177-1188.

23. Puértolas JA, Vadillo JL, Sánchez-Salcedo S, Nieto A, Gómez-Barrena E, Vallet-Regí M. Compression behaviour of biphasic calcium phosphate and biphasic calcium phosphate-agarose scaffolds for bone regeneration. Acta Biomater. 2011;7(2):841-847.

24. Gomes S, Renaudin G, Jallot E, Nedelec JM. Structural characterization and biological fluid interaction of Sol-Gel-derived $\mathrm{Mg}$-substituted biphasic calcium phosphate ceramics. ACS Appl Mater Interfaces. 2009;1(2):505-513.

25. Shanthini GM, Sakthivel N, Menon R, et al. Surface stiffening and enhanced photoluminescence of ion implanted cellulose - polyvinyl alcohol - silica composite. Carbohydr Polym. 2016;153:619-630.

26. Kinnear C, Burnand D, Clift MJ, Kilbinger AF, Rothen-Rutishauser B, Petri-Fink A. Polyvinyl alcohol as a biocompatible alternative for the passivation of gold nanorods. Angew Chem Int Ed Engl. 2014;53(46): 12613-12617.

27. Zhao YH, Zhang M, Liu NX, et al. The combined use of cell sheet fragments of periodontal ligament stem cells and platelet-rich fibrin granules for avulsed tooth reimplantation. Biomaterials. 2013;34(22): 5506-5520.

28. Bi L, Cheng W, Fan H, Pei G. Reconstruction of goat tibial defects using an injectable tricalcium phosphate/chitosan in combination with autologous platelet-rich plasma. Biomaterials. 2010;31(12):3201-3211.

29. Dohan EDM, Rasmusson L, Albrektsson T. Classification of platelet concentrates: from pure platelet-rich plasma (P-PRP) to leucocyteand platelet-rich fibrin (L-PRF). Trends Biotechnol. 2009;27(3): 158-167.

30. Wu CL, Lee SS, Tsai CH, Lu KH, Zhao JH, Chang YC. Platelet-rich fibrin increases cell attachment, proliferation and collagen-related protein expression of human osteoblasts. Aust Dent J. 2012;57(2): 207-212.

31. Dohan EDM, Bielecki T, Jimbo R, et al. Do the fibrin architecture and leukocyte content influence the growth factor release of platelet concentrates? An evidence-based answer comparing a pure platelet-rich plasma (P-PRP) gel and a leukocyte- and platelet-rich fibrin (L-PRF). Curr Pharm Biotechnol. 2012;13(7):1145-1152.

32. Linh NT, Lee KH, Lee BT. Functional nanofiber mat of polyvinyl alcohol/gelatin containing nanoparticles of biphasic calcium phosphate for bone regeneration in rat calvaria defects. J Biomed Mater Res A. $2013 ; 101(8): 2412-2423$. 
33. Nie L, Chen D, Suo J, et al. Physicochemical characterization and biocompatibility in vitro of biphasic calcium phosphate/polyvinyl alcohol scaffolds prepared by freeze-drying method for bone tissue engineering applications. Colloids Surf B Biointerfaces. 2012;100:169-176.

34. Bölükbaşı N, Yeniyol S, Tekkesin MS, Altunatmaz K. The use of platelet-rich fibrin in combination with biphasic calcium phosphate in the treatment of bone defects: a histologic and histomorphometric study. Curr Ther Res Clin Exp. 2013;75:15-21.

35. Jeong KI, Kim SG, Oh JS, et al. Effect of platelet-rich plasma and platelet-rich fibrin on peri-implant bone defects in dogs. J Biomed Nanotechnol. 2013;9(3):535-537.

36. Galleguillos-Silva R, Vargas-Hernández Y, Gaete-Garretón L. Wettability of a surface subjected to high frequency mechanical vibrations. Ultrason Sonochem. 2016;35(pt A):134-141.

37. Feng P, Peng S, Wu P, et al. A nano-sandwich construct built with graphene nanosheets and carbon nanotubes enhances mechanical properties of hydroxyapatite-polyetheretherketone scaffolds. Int $J$ Nanomedicine. 2016;11:3487-3500.

38. Anitua E, Sánchez M, Nurden AT, Nurden P, Orive G, Andía I. New insights into and novel applications for platelet-rich fibrin therapies. Trends Biotechnol. 2006;24(5):227-234.

39. Mahapatra C, Singh RK, Kim JJ, et al. Osteopromoting reservoir of stem cells: bioactive mesoporous nanocarrier/collagen gel through slow-releasing FGF18 and the activated BMP signaling. ACS Appl Mater Interfaces. 2016;8(41):27573-27584.

40. Li Y, Yang W, Li X, et al. Improving osteointegration and osteogenesis of three-dimensional porous Ti6A14V scaffolds by polydopamineassisted biomimetic hydroxyapatite coating. ACS Appl Mater Interfaces. 2015;7(10):5715-5724.

41. Zhang J, Guan J, Zhang C, et al. Bioactive borate glass promotes the repair of radius segmental bone defects by enhancing the osteogenic differentiation of BMSCs. Biomed Mater. 2015;10(6):065011.

42. Chen SH, Lei M, Xie XH, et al. PLGA/TCP composite scaffold incorporating bioactive phytomolecule icaritin for enhancement of bone defect repair in rabbits. Acta Biomater. 2013;9(5):6711-6722.

43. Wang L, Hu X, Ma X, et al. Promotion of osteointegration under diabetic conditions by tantalum coating-based surface modification on 3-dimensional printed porous titanium implants. Colloids Surf B Biointerfaces. 2016;148:440-452.

44. Costa DO, Prowse PD, Chrones T, et al. The differential regulation of osteoblast and osteoclast activity by surface topography of hydroxyapatite coatings. Biomaterials. 2013;34(30):7215-7226.

45. Jayasree A, Kuttappan S, Keyan KS, Nair MB. Evaluation of osteoinductive and endothelial differentiation potential of platelet-rich plasma incorporated gelatin-nanohydroxyapatite fibrous matrix. J Biomed Mater Res B Appl Biomater. 2016;104(4):771-781.

46. $\mathrm{Xu} \mathrm{W}$, Baribault $\mathrm{H}$, Adamson ED. Vinculin knockout results in heart and brain defects during embryonic development. Development. 1998; 125(2):327-337.

47. Xiu P, Jia Z, Lv J, et al. Tailored surface treatment of 3D printed porous Ti6A14V by microarc oxidation for enhanced osseointegration via optimized bone in-growth patterns and interlocked bone/implant interface. ACS Appl Mater Interfaces. 2016;8(28):17964-17975.

48. Man Y, Wang P, Guo Y, et al. Angiogenic and osteogenic potential of platelet-rich plasma and adipose-derived stem cell laden alginate microspheres. Biomaterials. 2012;33(34):8802-8811.

49. Fedorovich NE, Alblas J, Hennink WE, Oner FC, Dhert WJ. Organ printing: the future of bone regeneration. Trends Biotechnol. 2011; 29(12):601-606.

50. He HY, Zhang JY, Mi X, Hu Y, Gu XY. Rapid prototyping for tissueengineered bone scaffold by $3 \mathrm{D}$ printing and biocompatibility study. Int J Clin Exp Med. 2015;8(7):11777-11785.

51. Martínez-Vázquez FJ, Perera FH, Miranda P, Pajares A, Guiberteau F. Improving the compressive strength of bioceramic robocast scaffolds by polymer infiltration. Acta Biomater. 2010;6(11):4361-4368.
52. Rouwkema J, Rivron NC, van Blitterswijk CA. Vascularization in tissue engineering. Trends Biotechnol. 2008;26(8):434-441.

53. Karageorgiou V, Kaplan D. Porosity of 3D biomaterial scaffolds and osteogenesis. Biomaterials. 2005;26(27):5474-5491.

54. Liu M, Zheng H, Chen J, Li S, Huang J, Zhou C. Chitosan-chitin nanocrystal composite scaffolds for tissue engineering. Carbohydr Polym. 2016;152:832-840.

55. Wahl DA, Czernuszka JT. Collagen-hydroxyapatite composites for hard tissue repair. Eur Cell Mater. 2006;11:43-56.

56. Fonseca KB, Bidarra SJ, Oliveira MJ, Granja PL, Barrias CC. Molecularly designed alginate hydrogels susceptible to local proteolysis as three-dimensional cellular microenvironments. Acta Biomater. 2011; 7(4):1674-1682.

57. Hidalgo-Bastida LA, Cartmell SH. Mesenchymal stem cells, osteoblasts and extracellular matrix proteins: enhancing cell adhesion and differentiation for bone tissue engineering. Tissue Eng Part B Rev. 2010; 16(4):405-412.

58. Gómez S, Vlad MD, López J, Fernández E. Design and properties of 3D scaffolds for bone tissue engineering. Acta Biomater. 2016;42: 341-350.

59. Ekaputra AK, Prestwich GD, Cool SM, Hutmacher DW. The threedimensional vascularization of growth factor-releasing hybrid scaffold of poly (epsilon-caprolactone)/collagen fibers and hyaluronic acid hydrogel. Biomaterials. 2011;32(32):8108-8117.

60. Phipps MC, Xu Y, Bellis SL. Delivery of platelet-derived growth factor as a chemotactic factor for mesenchymal stem cells by bone-mimetic electrospun scaffolds. PLoS One. 2012;7(7):e40831.

61. Kanczler JM, Oreffo RO. Osteogenesis and angiogenesis: the potential for engineering bone. Eur Cell Mater. 2008;15:100-114.

62. de Gorter DJ, van Dinther M, Korchynskyi O, ten Dijke P. Biphasic effects of transforming growth factor $\beta$ on bone morphogenetic proteininduced osteoblast differentiation. J Bone Miner Res. 2011;26(6): $1178-1187$.

63. Poudel SB, Bhattarai G, Kim JH, et al. Local delivery of recombinant human FGF7 enhances bone formation in rat mandible defects. $J$ Bone Miner Metab. 2017;35(5):485-496.

64. Chiu YC, Fong EL, Grindel BJ, Kasper FK, Harrington DA, FarachCarson MC. Sustained delivery of recombinant human bone morphogenetic protein-2 from perlecan domain I - functionalized electrospun poly ( $\varepsilon$-caprolactone) scaffolds for bone regeneration. J Exp Orthop. 2016;3(1):25.

65. Mozdzen LC, Rodgers R, Banks JM, Bailey RC, Harley BA. Increasing the strength and bioactivity of collagen scaffolds using customizable arrays of 3D-printed polymer fibers. Acta Biomater. 2016;33:25-33.

66. Chen H, Qian Y, Xia Y, et al. Enhanced osteogenesis of ADSCs by the synergistic effect of aligned fibers containing collagen I. ACS Appl Mater Interfaces. 2016;8(43):29289-29297.

67. Wang Y, Jiang F, Liang Y, Shen M, Chen N. Human amnion-derived mesenchymal stem cells promote osteogenic differentiation in human bone marrow mesenchymal stem cells by influencing the ERK1/2 signaling pathway. Stem Cells Int. 2016;2016:4851081.

68. Lewallen EA, Jones DL, Dudakovic A, et al. Osteogenic potential of human adipose-tissue-derived mesenchymal stromal cells cultured on 3D-printed porous structured titanium. Gene. 2016;581(2):95-106.

69. Liu Y, Zhou Y, Feng H, Ma GE, Ni Y. Injectable tissue-engineered bone composed of human adipose-derived stromal cells and plateletrich plasma. Biomaterials. 2008;29(23):3338-3345.

70. Bose S, Roy M, Bandyopadhyay A. Recent advances in bone tissue engineering scaffolds. Trends Biotechnol. 2012;30(10):546-554.

71. He F, Ren W, Tian X, Liu W, Wu S, Chen X. Comparative study on in vivo response of porous calcium carbonate composite ceramic and biphasic calcium phosphate ceramic. Mater Sci Eng C Mater Biol Appl. 2016;64:117-123 
International Journal of Nanomedicine

Dovepress

\section{Publish your work in this journal}

The International Journal of Nanomedicine is an international, peerreviewed journal focusing on the application of nanotechnology in diagnostics, therapeutics, and drug delivery systems throughout the biomedical field. This journal is indexed on PubMed Central, MedLine, CAS, SciSearch ${ }^{\circledR}$, Current Contents ${ }^{\circledR} /$ Clinical Medicine,
Journal Citation Reports/Science Edition, EMBase, Scopus and the Elsevier Bibliographic databases. The manuscript management system is completely online and includes a very quick and fair peer-review system, which is all easy to use. Visit http://www.dovepress.com/ testimonials.php to read real quotes from published authors.

Submit your manuscript here: http://www.dovepress.com/international-journal-of-nanomedicine-journal 